\section{Q11.}

OAK RIDGE NATIONAL LABORATORY

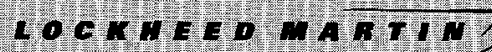

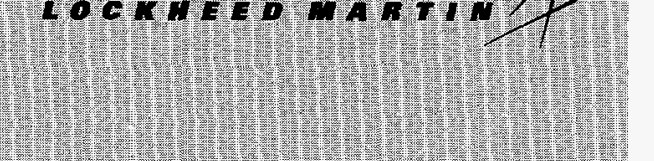

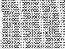

\section{(1)}

部

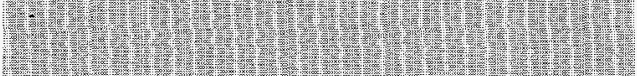

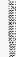

It)

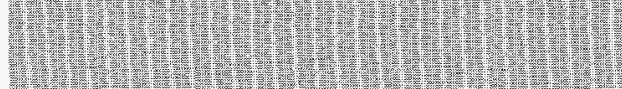

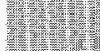

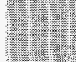

\section{(3)}

WAMAED AND OPERATED BY

LOCKHEED WAFNI ENERGY RESEARCH CORPORAJION TOR THE UNTED STATES

OEPARTUENT OF ENERCY

\section{RECEIVED SEP 141998 OSTI}

\section{Radio Frequency Current Drive Considerations for Small Aspect Ratio Tori}

\author{
M. D. Carter \\ E. F. Jaeger \\ D. J. Strickler \\ P. M. Ryan \\ D. W. Swain \\ D. B. Batchelor
}


This report has been reproduced directly from the best available copy.

Available to DOE and DOE contractors from the Office of Scientific and Technical Information, P.O. Box 62, Oak Ridge, TN 37831; prices available from (615) 576-8401, FTS 626-8401.

Available to the public from the National Technical information Service, U.S. Department of Commerce, 5285 Port Royal Rd., Springfield, VA 22161.

This report was prepared as an account of work sponsored by an agency of the United States Government. Neither the United States Government nor any agency thereof, nor any of their employees, makes any warranty, express or implied, or assumes any legal liability or responsibility for the accuracy, completeness, or usefuiness of any information, apparatus, product, or process disclosed, or represents that its use would not infringe privately owned rights. Reference herein to any specific commercial product, process, or service by trade name, trademark, manufacturer, or otherwise, does not necessarily constitute or imply its endorsement, recommendation, or favoring by the United States Govemment or any agency thereof. The views and opinions of authors expressed herein do not necessarily state or reflect those of the United States Government or any agency thereof. 


\section{DISCLAIMER}

\section{Portions of this document may be illegible in electronic image products. Images are produced from the best available original document.}


ORNL/TM-13576

Dist. Catagory UC-422

Fusion Energy Division

\title{
Radio Frequency Current Drive Considerations for Small Aspect Ratio Tori
}

\author{
M. D. Carter \\ E. F. Jaeger \\ D. J. Strickler \\ P. M. Ryan \\ D. W. Swain \\ D. B. Batchelor
}

Date Published: July 1998

\author{
Prepared by the \\ OAK RIDGE NATIONAL LABORATORY \\ Oak Ridge, Tennessee 37831 \\ managed by \\ LOCKHEED MARTIN ENERGY RESEARCH CORP. \\ for the \\ U.S. DEPARTMENT OF ENERGY \\ under contract DE-AC-05-96OR22464
}




\section{TABLE OF CONTENTS}

LIST OF FIGURES

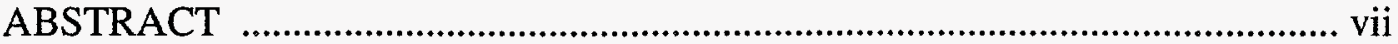

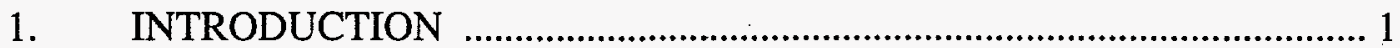

2. PLASMA COUPLING CONSIDERATIONS ....................................... 3

3. CURRENT DRIVE EFFICIENCY FOR HHFW USING DIFFERENT EQUILIBRIUM MODELS ................................................................... 5

4. ANALYSIS OF PRELIMINARY ANTENNA DESIGN FOR NSTX ....... 7

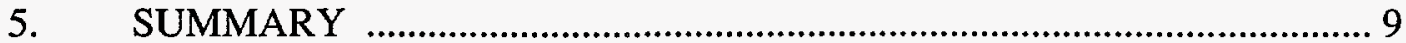

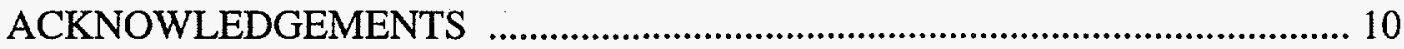

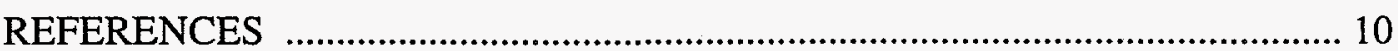

APPENDIX: VALIDITY OF THE RF MODELS IN THE HHFW REGIME .... 11 
$\checkmark$ 


\section{LIST OF FIGURES}

Figure

1. Magnetic fields in a spherical torus .......................................................... 15

2. Density profiles taken from FBEQ equilibria ............................................... 16

3. Power launch for uniform excitation in Fourier space ................................. 17

4. Poloidal magnetic field effect on parallel wavelength ................................... 18

5. Density and temperature profiles for different equilibria at $25 \% \beta$..............19

6. Magnetic topology for the FBEQ and Solov'ev equilibria at $25 \% \beta$...........19

7. The comparison between the FBEQ and Solov'ev equilibria at $5 \% \beta$....... 20

8. The magnetic topology for the FBEQ and Solov'ev at 5\% $\beta$...................... 20

9. Contours of HHFW current drive efficiency ................................................... 21

10. The RANT3D model for different poloidal orientations ............................... 22

11. Tilting the current straps produces a poloidal shift in the launched power spectrum 23

12. Fields from the RANT3D as boundary conditions for the PICES code ...... 24

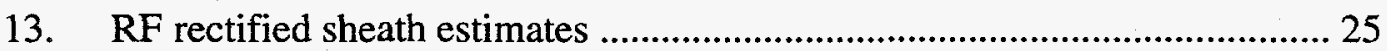

14. Warm plasma error relative to the hot ion calculation ................................... 26

15. The best approximation to the hot ion result uses unmagnetized ions ........ 26 


\title{
Radio Frequency Current Drive Considerations for Small Aspect Ratio Tori
}

\author{
M. D. Carter, E. F. Jaeger, D. J. Strickler, P. M. Ryan, \\ D. W. Swain, and D. B. Batchelor
}

\begin{abstract}
Noninductive current drive is required during plasma initiation and for current sustainment in the National Spherical Torus Experiment (NSTX). ${ }^{1}$ In this paper, the physics of high harmonic fast waves (HHFW) and the design of an antenna system for NSTX are considered using numerical models. For high current discharges in NSTX, the static magnetic field component in the poloidal direction varies widely during the discharge and can become comparable to the toroidal component in NSTX. Therefore, we calculate the plasma loading for a broad range of antenna and plasma geometries in a three-dimensional model, so that the results can be used to influence the antenna design. Two-dimensional calculations of the wave propagation and absorption in the core plasma indicate that the theoretical current drive efficiency for HHFW can be high, and a general survey of parameters gives a good target for the antenna design. The current drive efficiency calculation is sensitive to the equilibrium model because finite beta effects can substantially alter the calculation of the trapped particle fraction. Traditional methods of toroidally phasing an antenna array as well as poloidal phasing are studied to optimize the current drive efficiency for a range of equilibria. Non-zero poloidal mode excitation is also found to affect the antenna performance and flexibility. Performance expectations for a preliminary antenna design are given.
\end{abstract}




\section{INTRODUCTION}

Radio frequency (RF) power in the ion cyclotron range of frequencies is a useful tool for plasma heating and current drive that has not yet been extensively tested in a small aspect ratio torus. Noninductive current drive is required during plasma initiation and for current sustainment in the National Spherical Torus Experiment (NSTX), ${ }^{1}$ and computer modeling offers the only means to explore this regime at this time. In this paper, we review the RF options for NSTX that lead to the choice of high harmonic fast waves (HHFW). ${ }^{2}$. Then we use the RANT3D, ${ }^{3}$ GLOSI, $^{4}$ and PICES ${ }^{5}$ codes to analyze methods for optimizing plasma coupling and current drive efficiency in the HHFW regime. Both toroidal and poloidal antenna phasings are considered for launch from the equatorial plane on the outboard side of the device. The results have been found to be sensitive to assumptions in the plasma equilibrium, ${ }^{6}$ and finite $\beta$ effects, where $\beta$ is the ratio of plasma energy to magnetic energy, must be included to accurately calculate the trapped particle fraction and current drive efficiency. Preliminary antenna designs are studied and reasonable values are found for both the loading and driven steady-state current. Source terms for RF/edge interactions are also estimated.

Special consideration must be given in determining the accessibility of RF power in small aspect ratio devices, especially if the power is intended specifically for electrons. The usual methods with frequencies between the low-order ion cyclotron range of frequencies (LICRF) appear to be unfavorable because of the large variation in the static magnetic field strength, $B$, across the plasma cross section. However, as the plasma current becomes large, the poloidal magnetic field component can become comparable to the toroidal component, and a magnetic well can occur as illustrated schematically in Fig. 1. Thus, LICRF scenarios for electron current drive sustainment may be possible once a large steady-state current is achieved. At even lower frequencies, the Alfvén wave regime is also a possible candidate. At higher frequencies, the HHFW regime has been proposed. ${ }^{2}$

There are several practical disadvantages for driving currents in low frequency regimes in NSTX. One problem is the large size of the antenna required to launch the appropriate modes for strong electron damping. Another disadvantage is that a significant amount of current from some other source may be needed before LICRF power can be effectively delivered to electrons. Finally, the availability of high power generators for the low frequency regime would require a large added expense for NSTX.

The HHFW alternative can avoid excessive ion absorption if the majority ion beta, $\beta_{i}$, remains reasonably small, and no hot ion species are present. In this regime, a frequency of $30 \mathrm{MHz}$ is considered for NSTX so that ion absorption caused by finite $k_{\perp} \rho_{i}$ effects becomes small for moderate ion temperatures, where $k_{\perp}$ is the perpendicular wavenumber and $\rho_{i}$ is the ion gyroradius. HHFWs experience strong damping on the electrons for appropriately chosen phase velocities, and high current drive efficiency is possible. Current profile control may also be possible with HHFWs through toroidal and poloidal phase control of the antenna. For these reasons, and because of the availability of power in this frequency range, $6 \mathrm{MW}$ of HHFW power with phased array capability has been chosen as an initial system for the NSTX experiment. 
Regardless of the chosen RF method, the antenna design for current drive is complicated by several issues. First of all, the design must avoid damping by trapped electrons and simultaneously achieve reasonable plasma loading for a broad range of plasma parameters. Poloidal asymmetries in the plasma response caused by Hall currents can also affect the launch and deposition of the RF power. ${ }^{7,8}$ Finally, the large variation in the magnetic field angle near the antenna during a shot can complicate the design and give rise to RF edge-interactions for plasma facing components. ${ }^{9}$ Computer modeling is the only effective way to explore these issues for the NSTX antenna design.

The remainder of this paper is arranged as follows. In Sec. 2, we discuss plasma coupling considerations for the conditions anticipated in NSTX. Loading considerations and information guiding the choice of current strap angle are presented. The importance of the poloidal mode spectrum on loading is also discussed. In Sec. 3, we describe the plasma parameters and equilibria that are under consideration for NSTX and give general results from the PICES full wave code in full toroidal geometry. These results give an indication of which Fourier modes will produce the highest current drive efficiency from HHFWs and provide a target for designing an actual antenna. The results also illustrate the possibility of using poloidal antenna phasing to enhance flexibility, penetration, and current drive efficiency. The sensitivity of these results to finite beta effects in the equilibrium is also shown. In Sec. 4, we present results for the power spectra and the steady-state currents that might be expected from preliminary antenna designs under consideration for NSTX. Estimates for the RF fields at nearby conducting limiters are also given. A summary is given in Sec. 5. The validity of various physics approximations used in the RF models and numerical considerations are discussed in the Appendix for the parameters considered in this paper. 


\section{PLASMA COUPLING CONSIDERATIONS}

Launching HHFWs in NSTX suggests the use of compressional RF magnetic fields similar to conventional fast wave launchers. However, the large change in the magnetic field angle near the antenna during a shot complicates the choice for the antenna geometry. For high current, high $\beta$ operation, the poloidal component of the static magnetic field may even exceed the toroidal component near the antenna. Traditional fast wave antennas have been oriented so that the RF current is carried in the poloidal direction, which is nearly perpendicular to the static magnetic field throughout the discharge. The angle of the current straps cannot be changed during a shot, so a single fixed position must be chosen for NSTX. Alternatives to provide reasonable performance over a broad range of plasma conditions in NSTX include active poloidal phase control and/or choosing an optimal angle for the current strap tilt; however, cost constraints do not initially allow for active poloidal phase control in NSTX. The plasma response for these alternatives has been modeled using the RANT3D/GLOSI code combination.

The most obvious method to maintain the correct RF polarization in NSTX is to tilt the current straps at an intermediate angle that maintains reasonable polarization throughout the discharge. However, tilting the current straps of a phased array antenna has the added complication of modifying the Fourier spectrum in the poloidal direction. The peak in the poloidal spectrum for an infinite array of tilted current straps can be roughly estimated as

$$
\frac{m}{n} \approx-\frac{a}{R} \cot (\theta)
$$

where $m$ and $n$ are the poloidal and toroidal modes, respectively, $a$ is the average minor radius (the poloidal plasma circumference divided by $2 \pi$ ), $R$ is the major radius at the antenna, and $\theta$ is the angle of the current straps relative to the toroidal direction. For conventional antennas, $\theta=90^{\circ}$, so that the Fourier spectrum of the antenna current is centered at $m=0$. Using NSTX-like parameters of $R=1.58 \mathrm{~m}$ and $\mathrm{a}=0.88 \mathrm{~m}$, the Fourier spectrum for the RF current is centered near $m=n / 4$ for $\theta=66^{\circ}$. The width of the excited spectrum, $\Delta m$, in poloidal Fourier space is estimated by considering that a half-wavelength in the poloidal direction is roughly the poloidal extent of the current straps. This dimension is constrained in the NSTX design to be $\sim 1 \mathrm{~m}$, giving $\Delta m \approx 3$ for NSTX. Thus, several poloidal mode numbers must be considered to understand the effect of tilting on the plasma loading.

The GLOSI code is used to calculate the warm plasma response in a periodic sheared slab model of the tokamak edge, and this response is coupled to the three-dimensional RANT3D antenna loading code to determine the plasma coupling for different current strap orientations. As a preliminary step, a very small single strap antenna was considered to generate a nearly uniform excitation of Fourier modes in the plasma. We considered the density profiles shown in Fig. 2 for various finite $\beta$ equilibria in NSTX. The density profiles were extracted from the pressure profiles used for the equilibrium calculations by prescribing analytic temperature profiles. The temperature profiles were all taken to be parabolic with a maximum electron temperature of $1 \mathrm{keV}, 4 \mathrm{keV}$, and $5 \mathrm{keV}$ for the $5 \% \beta, 25 \% \beta$, and $40 \% \beta$ cases respectively. Maximum ion temperatures of $1 \mathrm{keV}, 1 \mathrm{keV}$, and $1.4 \mathrm{keV}$ were used for the $5 \% \beta, 25 \% \beta$, and $40 \% \beta$ cases, respectively. The minimum temperature in the scrape-off layer was $30 \mathrm{eV}$ for all cases and all species. The ion species mix was $5 \%$ hydrogen in deuterium. 
A delta function source is simulated using a $0.01-\mathrm{m}$-wide, $0.02-\mathrm{m}$-long strap carrying $1 \mathrm{~A}$ of RF current at $30 \mathrm{MHz}$. Results for current strap orientations of $\theta=90^{\circ}$ and $\theta=66^{\circ}$ are compared in Fig. 3. The static magnetic field direction at the antenna location is indicated for each case. Figure 3 clearly shows both a poloidal and toroidal asymmetry in the plasma response. This asymmetry is related to both the poloidal magnetic field and the Hall terms in the plasma dielectric. Loading is generally favored for co current drive except at very low toroidal mode numbers. Also, the poloidal width for good plasma response is $\Delta m \approx 3$ for $5 \% \beta$, increasing to $\Delta m \approx 5$ for the $40 \%$ $\beta$ case, and so the width of the plasma response is roughly the same at the width of the driven spectrum. As discussed in Sec. 3, the toroidal mode numbers of most interest for NSTX lie between $|n|=12$ and $|n|=4$ for good current drive efficiency over a broad range of $\beta$. Thus, the shift in the poloidal spectrum caused by a tilt of $\theta=66^{\circ}$ can range from 1 to 3 poloidal modes. Such a poloidal mode shift can substantially affect the loading depending on whether co (negative $n$ ) or counter (positive $n$ ) current drive is desired. The poloidal mode shift caused by tilting the straps is generally beneficial for counter current drive phasing, but it is detrimental for most co current drive phasings. For high $\beta$ cases, the plasma loading tends to be increased by the improved polarization of the tilted antenna, and the plasma response also shifts by enough to maintain good loading for $\theta=66^{\circ}$. However, for the $5 \% \beta$ case with co current drive, the tilt can reduce the loading by as much as a factor of 2 . This reduction in loading is caused by the shift of the poloidal spectrum of the source to a region where the plasma response is not optimal, and this reduction is not offset by an improvement in polarization. The reduction in loading occurs most prominently in precisely the desired range for NSTX co current drive operation, and may become important to the design if the RF system cannot compensate for the reduced loading. Figure 3 also clearly indicates that active poloidal phase control would be useful for optimizing the plasma loading.

Part of the physical explanation for the reduction in loading caused by tilting the antenna at low $\beta$ can be illustrated by considering the parallel wavenumber near the antenna as shown in Fig. 4. At low $\beta$, propagation in the edge region is weak because of the low plasma density and some tunneling through an evanescent layer is required. The high parallel conductivity of the plasma allows modes with long parallel wavelengths to penetrate through the evanescent layer more easily than those with shorter parallel wavelengths. At high enough $\beta(\sim 25 \%)$ the Shafranov shift moves higher density plasma closer to the antenna, thereby reducing the evanescent layer for the modes of interest, and polarization eventually becomes the dominant effect.

The conclusion from these results is that plasma loading could be best optimized by actively controlling both the toroidal and poloidal phase of the antenna array; however, such a system might be technically difficult and expensive to build. Improvement in the polarization can be obtained by tilting the current straps, but the tilt introduces a poloidal shift in the power spectrum that can significantly reduce the loading in co current drive scenarios for low $\beta(5 \%)$ operation. Without active poloidal phase control to compensate for this shift, a trade-off in antenna performance at low $\beta$ versus high $\beta$ must be considered in determining the strap orientation. This trade-off may also affect the antenna control system because the decoupling circuitry may become more complicated for low plasma loading. 


\section{CURRENT DRIVE EFFICIENCY FOR HHFW USING DIFFERENT EQUILIBRIUM MODELS}

In addition to accessibility, the launched power must provide a large fraction of the total steady state current in NSTX throughout the discharge. In this section, we use the two-dimensional RF model of the core plasma, PICES, to calculate the power deposition. We estimate the steady state current that can be driven by HHFWs in NSTX using the Ehst/Karney empirical fit, accounting for trapped electrons at the location where the power is absorbed. ${ }^{10}$ Although this method for calculating current drive is not as accurate as an adjoint method, it does provide a relative indication of the current drive performance. ${ }^{11}$ A single poloidal and toroidal mode for the $R F$ is imposed as a boundary condition on the last closed flux surface. The imposed mode is then scanned in poloidal and toroidal mode space to help find the modes that produce optimal current drive efficiency. These results are used to help determine the operational capability that must be designed into the antenna system.

The results of RF calculations in the plasma core have been found to be very sensitive to the equilibrium parameters that are chosen. ${ }^{6}$ Thus, we use the most accurate, self-consistent, numerical calculations of finite $\beta$ equilibria that are available for NSTX from the free-boundary MHD equilibrium code FBEQ. ${ }^{12}$ The NSTX equilibria are constrained by a fixed major radius of 0.854 $\mathrm{m}$, aspect ratio of 1.26 , toroidal field of $0.3 \mathrm{~T}$, total current of $1 \mathrm{MA}$, elongation of 2.0 , and internal inductance of 0.2 . Three representative values of total $\beta, 5 \%, 25 \%$, and $40 \%$, are considered. A temperature profile is assumed and the density profile is calculated using the same pressure profile used in the equilibrium calculation.

We also contrast these results with those from a variation of the Solov'ev equilibrium model in an attempt to isolate the parameters that produce the most sensitivity. The comparison shows that much better agreement between the different equilibria can be obtained than was found in Ref. 6 if care is taken to use similar plasma shapes, and comparable density and temperature profiles. However, the Solov'ev equilibrium cannot accurately treat trapped electron effects because of finite $\beta$ corrections to the magnetic field. This inaccuracy can lead to a reduction in predicted efficiency by as much as a factor of 2 for similar plasma profiles. The results for $5 \%$ and $25 \% \beta$ cases are considered to illustrate these results.

In the $25 \% \beta$ case, the magnetic axis for the Solov'ev equilibrium was placed in the same location as the FBEQ result to partly account for the Shafranov shift. The q-profile in the Solov'ev model was adjusted to make the angle of the magnetic field at the antenna location nearly equal to that of the finite $\beta$ result. A comparison of the temperature and density profiles at $25 \% \beta$ for the two equilibria is shown in Fig. 5; the flux surfaces and magnetic field strength are shown in Fig. 6 . The comparison shows that the plasma parameters are very similar, except that the magnetic field strength is modified by finite $\beta$ effects.

The 5\% $\beta$ case using the Solov'ev equilibrium was complicated by the magnetic axis location in the FBEQ equilibrium, $0.96 \mathrm{~m}$, and the lack of triangularity in the Solov'ev model. Again, the q-profile was chosen so that the magnetic field components were nearly the same on the outboard side near the equatorial plane. However, the analytic equilibrium would not close on the inside of the tokamak without simultaneously extending the position of the magnetic axis to $\sim 1.1 \mathrm{~m}$ for the 
Solov'ev equilibrium (similar to the $25 \% \beta$ case). A comparison of the plasma parameters for the $5 \% \beta$ case is shown in Fig. 7, and the flux surfaces and magnetic field strength are shown in Fig. 8 .

A crude indication of the theoretical limit for current drive efficiency is obtained by exciting one pure toroidal and poloidal mode at a time on the plasma surface in PICES and then scanning the excited surface mode. Figure 9 shows the current drive efficiency results from scans for the $5 \%$ and $25 \% \beta$ equilibria previously described. The crowding of lines near toroidal mode zero denotes the rapid change from positive to negative current drive.

The results for FBEQ equilibria in Fig. 9 indicate the modes that should be produced by the antenna to achieve the best current drive efficiency at different $\beta$ values. To maintain optimum current drive efficiency at $30 \mathrm{MHz}$ for the geometries considered here, the antenna should excite toroidal mode numbers near $n=-8$ for low to moderate $\beta$, and then shift to $n=-4$ to $n=-2$ as the $\beta$ increases. Note that a real antenna will produce a spread of toroidal modes, and a rapid change in efficiency is possible for very small $n$ numbers because some of the power may drive current in the direction opposite to what is desired.

The poloidal dependence of the current drive efficiency may also be an issue for the antenna design. A shift in the poloidal spectrum by a few modes can change the efficiency by roughly $20 \%$. The poloidal shift caused by tilting the current straps is detrimental to the efficiency for both co and counter current drive. Again, this poloidal mode behavior can be partially attributed to the launched parallel wavelength of the wave as described in Fig. 4. Waves with longer parallel wavelengths can penetrate further into the plasma before the phase speed becomes resonant with the bulk of the electron distribution. As in the case of plasma loading, a drop in current drive efficiency for tilted straps may be acceptable if the antenna can meet its design objectives. The total current expected for preliminary designs will be presented in Sec. 4 .

The results of Fig. 9 also show that the Solov'ev equilibria predict a current drive efficiency that is as much as a factor of 2 lower than the FBEQ results. Since differences in the plasma profiles have been largely excluded, we attribute most of the difference to the lack of finite $\beta$ effects in the Solov'ev equilibria, and the consequent errors in the calculation of the trapped electron fraction. Thus, numerical MHD calculations representing realistic plasma equilibrium scenarios are required for accurate modeling of the current drive in NSTX 


\section{ANALYSIS OF PRELIMINARY ANTENNA DESIGNS FOR NSTX}

A preliminary antenna design for NSTX uses 12 evenly spaced current straps driven at $30 \mathrm{MHz}$. The design can operate with toroidal phasings between straps, $\Delta \phi$, of $30^{\circ}, 60^{\circ}$, and $90^{\circ}$ to excite spectra that are peaked near the antenna at $n=-4,-8$, and -12 , respectively. Initially, plans call for a fixed $180^{\circ}$ phase shift to be placed between straps 1 and 7,2 and 8 , etc. (see Fig. 10), to simplify the RF circuit and to provide the best spectrum with $\Delta \phi=30^{\circ}$ for high $\beta$ current drive operation. Other phasings that would be more suitable for heating rather than current drive will also be possible. Poloidal phase control is not anticipated for the initial system because of the added expense and complexity required in such a system. However, the port locations for the initial design may make it possible to tilt the current straps, permitting strap angles of $\theta=90^{\circ}, \theta=78^{\circ}$, or $\theta=66^{\circ}$.

In this section, we will investigate the current drive and overall performance for preliminary designs with two orientations for the current straps for the $\beta=5 \%$ and $25 \%$ scenarios. The calculations are performed using combinations of the RANT3D model with both the GLOSI (slab) and PICES (toroidal) core plasma models. We will also present a crude analysis of the rectified RF voltages that might be expected on conducting limiters near the antenna. The model results show that tilting the current straps has a minimal effect on current drive efficiency, and that the poloidal shift in the spectrum reduces the loading at $5 \% \beta$ as expected from the results in Sec. 2. The near-field analysis shows that the rectified fields are larger for the $40 \% \beta$ case than for the $5 \% \beta$ case, and that tilting the current straps does not significantly change the maximum rectified voltages, although it may change the location of local maxima.

In Fig. 10, we show the RANT3D model for preliminary designs with strap angles of $90^{\circ}$ and $66^{\circ}$. The antenna is bounded in the poloidal direction by MHD stabilizing plates. The conducting boundaries in the toroidal direction are not yet well defined and have been arbitrarily located in the RANT3D models. Insulating material (boron nitride) will be used to limit the diffusion of plasma into the antenna, and the RANT3D model assumes vacuum behind these limiters. Additional insulating layers may also be positioned to further protect conducting limiters from RF rectification effects.

The effect that tilting the current straps has on plasma loading is similar to what is expected from the earlier delta-function antenna analysis in Sec. 2 . The power spectra for the $5 \%$ and $40 \% \beta$ cases are shown in Fig. 11. The spectrum for the $\Delta \phi=60^{\circ}$ is split because straps 1 and 7,2 and 8 , and so on, are locked $180^{\circ}$ out of phase to simplify the RF circuit design. The increase in the poloidal shift with larger toroidal mode numbers, as given by Eq. 1, is clearly seen. The tilt reduces the loading at $5 \% \beta$, but increases the loading at $40 \% \beta$.

Profiles of power and current drive for the preliminary antenna designs can be calculated by using the electric field results on the first wall from the RANT3D/GLOSI calculation as a boundary condition to the PICES code. The results of these calculations for the $5 \% \beta$ case (FBEQ in Fig. 8) with $\Delta \phi=60^{\circ}$, and the $25 \% \beta$ case (FBEQ in Fig. 6) with $\Delta \phi=30^{\circ}$ are shown in Fig. 12 for a strap orientation of $\theta=90^{\circ}$. Very similar profiles are obtained for these cases with straps oriented at $\theta=66^{\circ}$. Penetration to the center of the plasma becomes more difficult for the high $\beta$ cases, causing the current drive efficiency to drop for the $25 \% \beta$ case. 
The total current driven by $\sim 6 \mathrm{MW}$ of HHFW power in the model for $5 \% \beta$ with $\Delta \phi=60^{\circ}$ is $420 \mathrm{kA}$ for $\theta=90^{\circ}$ straps and $390 \mathrm{kA}$ for straps oriented at $\theta=66^{\circ}$. With a $\Delta \phi=30^{\circ}$, the $5 \% \beta$ case generates roughly $390 \mathrm{kA}$ regardless of the strap tilt. The total current driven in the model by $\sim 6 \mathrm{MW}$ for the $25 \% \beta$ case is $300 \mathrm{kA}$ for straps with $\theta=90^{\circ}$ and $320 \mathrm{kA}$ for straps with $\theta=66^{\circ}$. Thus, the current drive efficiency in the model is not as sensitive as the loading to the strap tilt. Note also that the total driven current for the $5 \% \beta$ case can be increased to $570 \mathrm{kA}$ at $6 \mathrm{MW}$ for $\Delta \phi=60^{\circ}$ if the lock between arrays, straps 1 and 7,2 and 8 , etc., is changed from $180^{\circ}$ to $0^{\circ}$. However, changing the locking between arrays to $0^{\circ}$ spoils the spectrum for $\Delta \phi=30^{\circ}$, and reduces the current for the high $\beta$ cases.

Another issue that may impact the antenna design is the effect of RF near-field plasma excitation. We use the RANT3D code to estimate the plasma currents and parallel electric fields near conducting limiters in the model. These field calculations can be used to check the possible benefits that tilting the current straps might have on RF/edge interactions. The results indicate that RF charge builds especially on the upper poloidal protection and also some on the toroidal protection. We use the loading results from the RANT3D calculation to normalize the electrostatic fields near the limiters for $6 \mathrm{MW}$ of delivered power for two tilt geometries at $5 \% \beta$ and $40 \% \beta$ and estimate the rectified voltage.

Because the RF fields at the conducting boundaries are dominated by surface charge, the fields are localized very near the conducting surface even in the vacuum region of the model. The local rectified voltage estimate is simplified by the fact that these fields decay rapidly along the field line. Following Ref. 9, the parallel electric field should properly be integrated along the entire length of the field line, but a local calculation at each end gives a good indication of the maximum rectified voltage. The local RF voltage is estimated by integrating along the magnetic field lines near each end, which gives the dominant contribution. The DC rectified voltage is estimated from the RF voltage by multiplying by a rectification factor of $0.6 .^{9}$ The results of the calculations are shown in Fig. 13 where the shading at the conducting limiters is proportional to the rectified voltage, and values of local maxima are labeled. The results should be considered to be a crude, and probably pessimistic estimate, but show that the rectified voltage in the $40 \% \beta$ case may reach almost $4 \mathrm{kV}$. The rectified voltage for the $40 \% \beta$ scenario is much higher than for the $5 \% \beta$ case, probably because larger RF-induced plasma currents flow closer (radially) to the conducting limiters; as shown by the density profiles near the edge in Fig. 2 . 


\section{SUMMARY}

The RANT3D, PICES, and GLOSI codes have been used to model high harmonic fast wave scenarios for preliminary antenna designs in NSTX. Plasma loading, current drive efficiency, and sources for nonlinear RF sheath effects have been estimated for scenarios with $1 \mathrm{MA}$ of current and 3 different values of $\boldsymbol{\beta}$. A comparison of results based on Solov'ev equilibria and numerical equilibria for NSTX shows that the current drive efficiency is sensitive to the details of the plasma equilibrium representation. Therefore, the HHFW calculations have been performed with equilibria generated by the free-boundary code, FBEQ.

Poloidal effects in the plasma response, caused by the steep density gradients and the large poloidal magnetic fields expected in NSTX, can significantly affect the antenna performance. These poloidal effects become important when considering the phasing and orientation of the current straps for the antenna. Full control of both the toroidal and poloidal phase of the antenna is desirable, and possible for future antenna designs. However, cost constraints for the initial design allow only for the possibility of tilting the current straps away from the traditional poloidal orientation by as much as $24^{\circ}$. Tilting the current straps can improve the wave polarization; however, the tilt also introduces a poloidal phase shift in the launched power spectrum that depends on the toroidal phasing. This poloidal shift reduces the loading for the $5 \% \beta$ scenario despite the improved polarization because the waves must penetrate through an evanescent layer that favors longer parallel wavelengths (see Fig. 4). Tilting the straps increases the loading for the $40 \% \beta$ scenario because there is only a small evanescent layer, and the improved polarization is beneficial in that case.

A reduction in loading at low $\beta$ may be of concern because the RF circuit may require a more complicated decoupling system if the loading becomes too low, and all scenarios must pass though a low $\beta$ regime at some point during the discharge. Some improvement in the loading at low $\beta$ for a tilted strap design might be obtained by shifting the plasma nearer the antenna during low $\beta$ phases of the discharge if a tilt is implemented in the actual design.

Current drive efficiency is not strongly affected by tilting the current straps for $\beta$ greater than roughly $5 \%$ because the plasma $\beta$ tends to help align $|\mathrm{B}|$ with flux surfaces to reduce trapped particle effects. If the straps are tilted away from a poloidal orientation, then the resulting poloidal shift in the spectrum can lower the current drive efficiency in the $5 \% \beta$ scenario during co current drive operation. This loss in efficiency becomes more pronounced for slower waves because the poloidal shift in the spectrum caused by the strap tilt is roughly proportional to the toroidal mode number. The analysis using the Ehst/Karney fit for efficiency shows that the preliminary design should drive $-400 \mathrm{kA}$ for $6 \mathrm{MW}$ of HHFW input power in the scenarios considered. These estimates may be conservative based on work that uses an adjoint method for the current drive calculation."

RF sheath estimates indicate that tilting the current straps does not significantly change the RF sheath voltage. RF sheath estimates are much larger for the $40 \% \beta$ case than for the $5 \% \beta$ case reaching $-4 \mathrm{kV}$ in the $40 \% \beta$ scenario and less than half that value for the $5 \% \beta$ case. The rectification appears to be the largest at the top conducting boundary for the full current field geometries considered here regardless of the strap orientation. Boron nitride limiters and 
protection for nearby conducting surfaces are planned to protect the antenna structure from these RF voltages.

The modeling shows that reasonable performance can be obtained from the preliminary antenna design. Based on the models, designs, and full current scenarios considered here, tilting the current straps away from the poloidal orientation may be beneficial for very high $\beta$ scenarios, but may reduce performance for low $\beta(5 \%)$ operation. Additional RF control circuitry or plasma position control may be needed if the loading becomes too low during low $\beta$ phases of high current discharges. An RF experiment with different strap orientations would be desirable for comparison with the modeling results presented in this paper. Future modeling work to implement generalized $Z$ functions and to enhance the poloidal resolution in the PICES full wave code is desirable. A two-dimensional, full-wave, hot-plasma analysis is also desirable, but such a model is well beyond the present state-of-the-art.

\section{Acknowledgments}

The antenna design for NSTX has been a team effort with the RF design team at the Princeton Plasma Physics Laboratory including R. Majeski, R. Wilson, and M. Ono. This work has been sponsored by the Oak Ridge National Laboratory managed by Lockheed Martin Energy Research Corporation for the U.S. Department of Energy under Contract Number DE-AC05-96OR22464.

\section{References}

1. J. Robinson et al., Bull. Am. Phys. Soc. 39, 1573 (1994) .

2. M. Ono, Phys. Plasmas 2, 4075 (1995).

3. M. D. Carter et.al., Nucl. Fusion 36, 209 (1996).

4. C. Y. Wang et al., Phys. Plasmas 2, 2760 (1995).

5. E. F. Jaeger, D. B. Batchelor, and D. C. Stallings, Nucl. Fusion 33, 179 (1993).

6. M. D. Carter et al., 12th Topical Conference on Radio Frequency Power in Plasmas, AIP Conference Proc. 403, eds. P. M. Ryan and T. Intrator, p 247.

7. E. F. Jaeger et al., Nucl. Fusion 38, 1 (1998).

8. R. I. Pinsker et al., Nucl. Fusion 26, 941 (1986).

9. J. R. Myra, D. A. Dippolito, and M. J. Gerver, Nucl. Fusion 30, 845 (1990).

10. D. A. Ehst and C. F. F. Karney, Nucl. Fusion 31, 1933 (1991).

11. J. C. Wright, C. K. Phillips, and P. T. Bonoli, 12th Topical Conference on Radio Frequency Power in Plasmas, AIP Conference Proc. 403, eds. P.M. Ryan and T. Intrator, p. 273.

12. D. J. Strickler et al., Fusion Tech. 19, 1452 (1991). 


\section{APPENDIX. VALIDITY OF THE RF MODELS IN THE HHFW REGIME}

The full wave models used in this paper are subject to several limitations that must be considered before the results can be interpreted. These limitations can be categorized into numerical convergence issues, and plasma physics approximations that are fundamental to the algorithms. In this section, we briefly discuss the validity of the RANT3D/GLOSI model for launching the power through the plasma edge region. Next, we consider convergence and resolution issues for the PICES code. Finally, we consider the validity of the warm plasma approximation for plasma regimes that are appropriate for NSTX.

The 1D GLOSI code does not retain the full toroidal geometry needed to accurately calculate the power deposition, but it does provide a reasonable model in the plasma edge region. In these simulations, we use a linearly sheared magnetic field in GLOSI with poloidal and toroidal components at the antenna appropriate for the discharge equilibrium. The edge region near the plasma has a reasonably low temperature, and the warm plasma approximation should remain valid for several perpendicular wavelengths away from the antenna. Thus, the launched power spectrum and the RF near-fields can be reasonably modeled in three dimensions using the RANT3D code with a plasma impedance relationship calculated from the GLOSI results. The GLOSI model for the impedance assumes that power penetrating to the magnetic axis does not return to the antenna. This approximation is reasonable so long as the system geometry does not reflect significant power back to the antenna after the waves pass beyond the magnetic axis.

For the PICES code, reasonable numerical convergence in the power deposition is obtained for the dominant low-order poloidal modes without fully resolving the shortest wavelength modes in the system. However, the ability to fully resolve the poloidal structure of waves in the system depends upon the boundary conditions that are applied. Aside from the actual boundary condition at the vacuum vessel wall, an additional approximation for the direction of $k_{\perp}$ in the reduced order approximation can influence the convergence near the magnetic axis. ${ }^{1}$ Reasonable convergence properties are found for NSTX parameters when the $k_{\perp}$ value for the reduced order terms is taken to be in the radial direction. If the value for $k_{\perp}$ is allowed to have a poloidal component, then the convergence can become much more difficult because the poloidal wavelength of the waves can become comparable with the maximum poloidal resolution allowed by most available computational resources. This approximation is reasonable if most of the power is absorbed before reaching the origin. It may also give reasonable results if the absorption is reasonably weak as in the case of low $\beta_{i}$, where $\beta_{i}$ is the ion $\beta$. Thus, for the results presented here, the reduced order approximation is implemented with the assumption that $k_{\perp}$ is in the radial direction.

The actual boundary at the vacuum vessel wall can also lead to convergence problems for the waves in the two-dimensional plasma models. A realistic antenna source can introduce poloidal modes that become too small to resolve with the present implementation of the PICES code, especially if these modes propagate and subsequently turn in the poloidal direction. This problem is particularly difficult on the outer flux surfaces because of the natural spreading of the poloidal grid in flux coordinates. However, applying a single fixed mode at the plasma boundary can stabilize the calculation. When the single mode chosen at the plasma boundary is systematically scanned, reasonable guidance is provided for the antenna design to help optimize the current drive 
efficiency. Power deposition profiles can also be reasonably well converged if only the dominant low-order poloidal modes are retained in a more realistic antenna model. Thus, results from relatively low resolution runs of the PICES code can provide useful information about the HHFW current drive to guide the antenna design and to develop operational scenarios.

Future enhancements in the PICES algorithm and improvements in the matrix solution method are possible to further explore the numerical resolution issues for plasma calculations in toroidal geometry. However, decisions for the NSTX antenna design require that we use results from our present algorithms as well as possible. Thus, to avoid some of the numerical difficulties, we present results from the PICES code using systematic scans of fixed poloidal modes at the outer plasma boundary and low resolution models for the antenna, and we assume a radial direction for $k_{\perp}$ in the reduced order approximation.

The most obvious assumption in the physics of both the PICES and GLOSI codes for NSTX-like parameters is the warm plasma approximation, especially in light of the fact that the perpendicular wavelength can become comparable to the ion gyroradius. Other assumptions, such as the use of simple, as opposed to generalized $Z$ functions, ${ }^{2,3}$ are expected to be reasonable so long as the ion absorption remains small compared with the electron absorption.

The warm plasma approximation assumes that the product of the perpendicular wave number and the gyroradius $k_{\perp} \rho_{i}$, remains small, and only second-order terms are retained in a $k_{\perp} \rho_{i}$ expansion. Although this assumption is valid for electrons in the HHFW regime, it becomes suspect for ions in NSTX because $k_{\perp} \rho_{i} \approx\left(\omega v_{t i}\right) /\left(\Omega_{i} v_{a}\right) \sim \omega \beta_{i}^{1 / 2} / \Omega_{i}$ where $\omega$ is the RF frequency, $v_{t i}$ is the ion thermal speed, $v_{a}$ is the Alfvén speed, $\beta_{i}$ is the ion $\beta$, and $\Omega_{i}$ is the ion cyclotron frequency. Thus, we check the validity of the warm ion approximation for NSTX-like parameters by comparing the dispersion relation obtained from a full hot plasma dielectric with that obtained with the warm ion approximation. Note that for fixed $\beta_{i}$, finite ion gyroradius effects can be reduced by either lowering $\omega$ or increasing $|\boldsymbol{B}|$ so long as low order resonances are avoided.

The plasma dielectric tensor can be written as

$$
\varepsilon_{j k}=\delta_{j k}+\frac{i}{\varepsilon_{0} \omega} \sum_{s} \sigma_{j k}^{s}
$$

where $\delta_{j k}$ is 1 for $j=k$ and 0 for $j \neq k, \varepsilon_{0}$ is the permittivity of free space, and $\sigma_{j k}^{s}$ is the conductivity for each plasma species, $s$. For the most accurate solution in magnetized plasma, $\sigma_{j k}^{s}$ in Eq. A1 is calculated using a hot plasma model with 40 Bessel functions retained. In the warm plasma approximation, it is assumed that $k_{\perp} \rho_{i} \ll<1$, and the Bessel functions are expanded retaining terms having order up to and including $\left(k_{\perp} \rho_{i}\right)^{2}$. The conductivity of the ions may also be reasonably approximated in an unmagnetized limit with finite ion temperature because the ion gyroradius can be large compared with the wavelength for HHFW at these parameters. ${ }^{4}$

The accuracy in calculating the real and imaginary parts of $k_{\perp}$ using various ion orbit approximations is illustrated in Fig. 14 for high $\beta_{i}$ conditions at $41 \mathrm{MHz}$ with NSTX-like parameters. The magnetic field was chosen to have a simple $1 / R$ scaling to give a more pessimistic result concerning the finite $k_{\perp} \rho_{i}$ effects on the outside of the tokamak than if the poloidal 
component of the magnetic field were added. The calculation also assumes that no population of hot ions from beams or other hot ion sources exist. The case with hot, unmagnetized ions gives the best approximation to the hot ion dispersion relation with small oscillatory errors near each ion resonance. The case of warm magnetized ions gives substantially reduced values for the imaginary part of $k_{\perp}$ for these high $\beta_{i}$ conditions. However, the warm approximation is reasonably accurate for more modest values of $\beta_{i}$.

Figure 14 shows the errors in the real and imaginary parts of $k_{\perp}$ caused by using the warm plasma approximation instead of considering hot ions. Clearly, the warm plasma model used by the GLOSI and PICES codes can underestimate the wave damping if $k_{\perp} \rho_{i}$ in NSTX is too large. However, the results in Fig. 15 show that the error introduced by using a warm ion approximation is at worst $\sim 10 \%$, and less than $5 \%$ on average if the ion temperature is kept below $1 \mathrm{keV}$ for a peak density of $5 \times 10^{19} \mathrm{~m}^{-3}$. For the same density and a $2 \mathrm{keV}$ ion temperature, the warm approximation is unacceptable with more than $30 \%$ error in the damping scale-length. For a lower peak density of $3 \times 10^{19} \mathrm{~m}^{-3}$ and an ion temperature of $2 \mathrm{keV}$, the warm approximation systematically underestimates the damping by only about $10 \%$. Thus, we conclude that the PICES and GLOSI models can be used with reasonable confidence for deuterium plasmas with magnetic field parameters similar to those of NSTX $\left(0.32 \mathrm{~T}\right.$ on axis) with frequencies below $41 \mathrm{MHz}$ and $n_{i} T_{i}<$ $6 \times 10^{19} \mathrm{keVm}^{-3}\left(\beta_{i}<12 \%\right)$. The PICES model further includes absorption caused by hot ion species by using the Bessel functions, a reasonable approximation so long as the hot ion species do not strongly affect the wave propagation (real part of $k_{\perp}$ ).

\section{References}

1. E. F. Jaeger et al., Nucl. Fusion 38, 437 (1998).

2. D. Smithe et al., Phys. Rev. Letters 60, 801 (1988).

3. M. Brambilla, Phys. Letters A 188, 376-383 (1994).

4. D. B. Batchelor et al., "High Harmonic Ion Cyclotron Heating and Current Drive in Ultra Small Aspect Ratio Tokamaks," Fusion Energy 1996, Proceedings of the 16th International Atomic Energy Agency (IAEA) Fusion Energy Conference, Montreal, Canada, October 7-11, 1996 (IAEA, Vienna, 1997), Vol. 2, pp 675-681. 


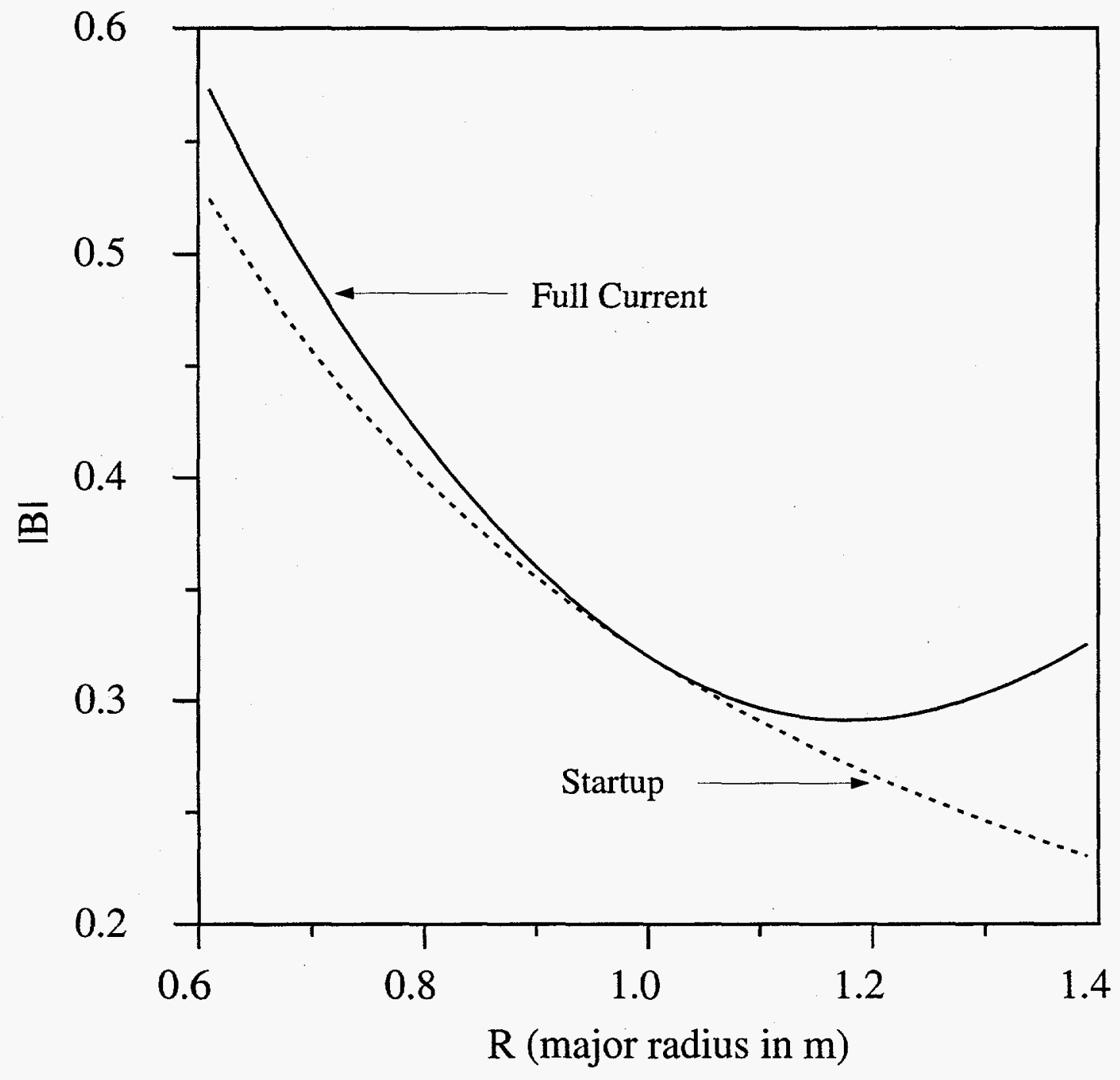

Fig. 1. Large plasma currents and low toroidal magnetic fields in a ST lead to a field profile that is not typical of tokamaks with larger aspect ratios. Low-order ion cyclotron resonances may be accessible after the poloidal field from the plasma current is establisted. 


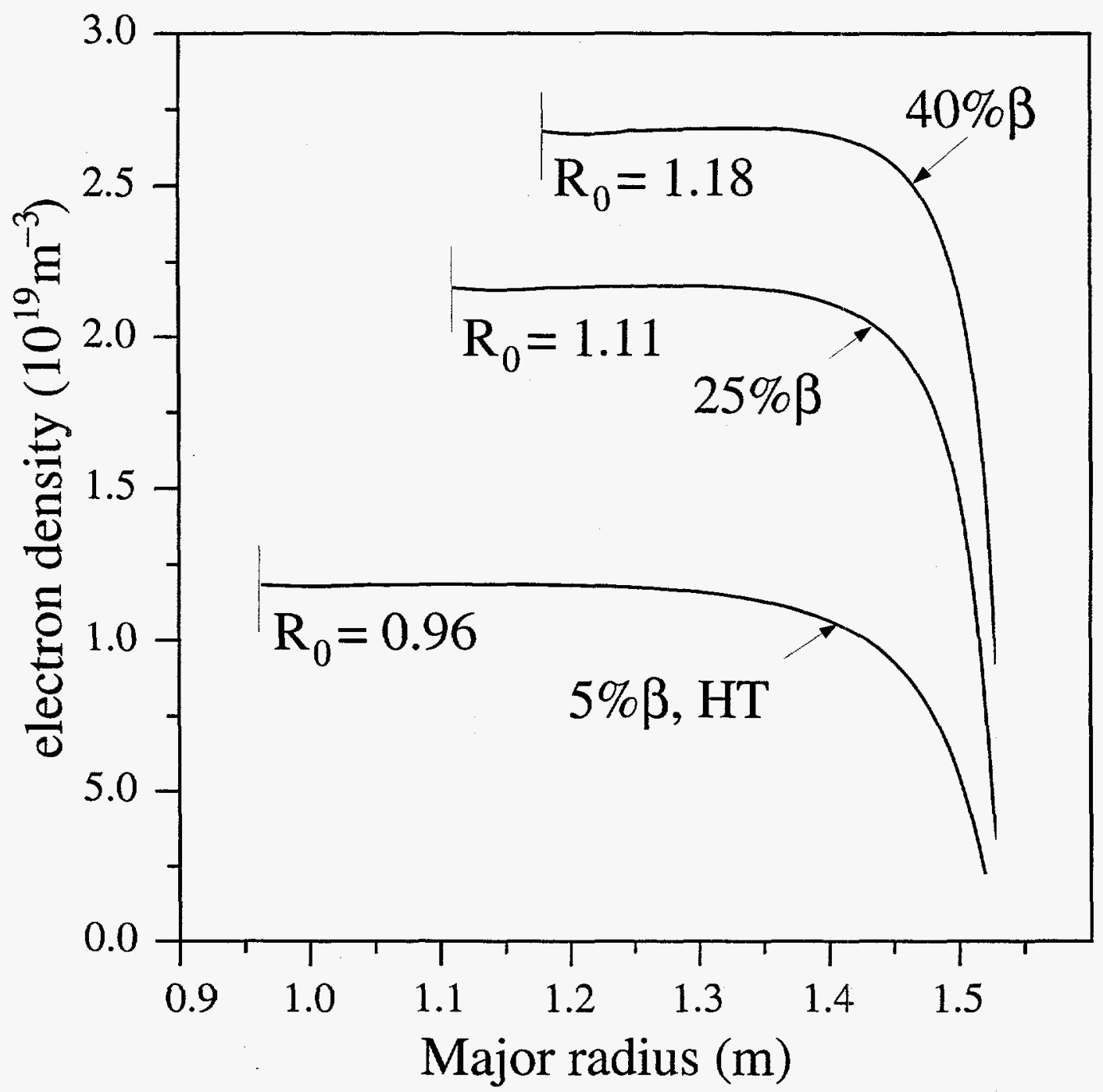

Fig. 2. Density profiles taken from FBEQ equilibrium files for loading studies. The scrape-off layer was extended $0.05 \mathrm{~m}$ beyond the last closed flux surface and an exponential with $0.01 \mathrm{~m}$ scale length was used to extend the density from the location where the density is $10 \%$ of the maximum density. 
Impulse source, $0^{\circ}$ tilt
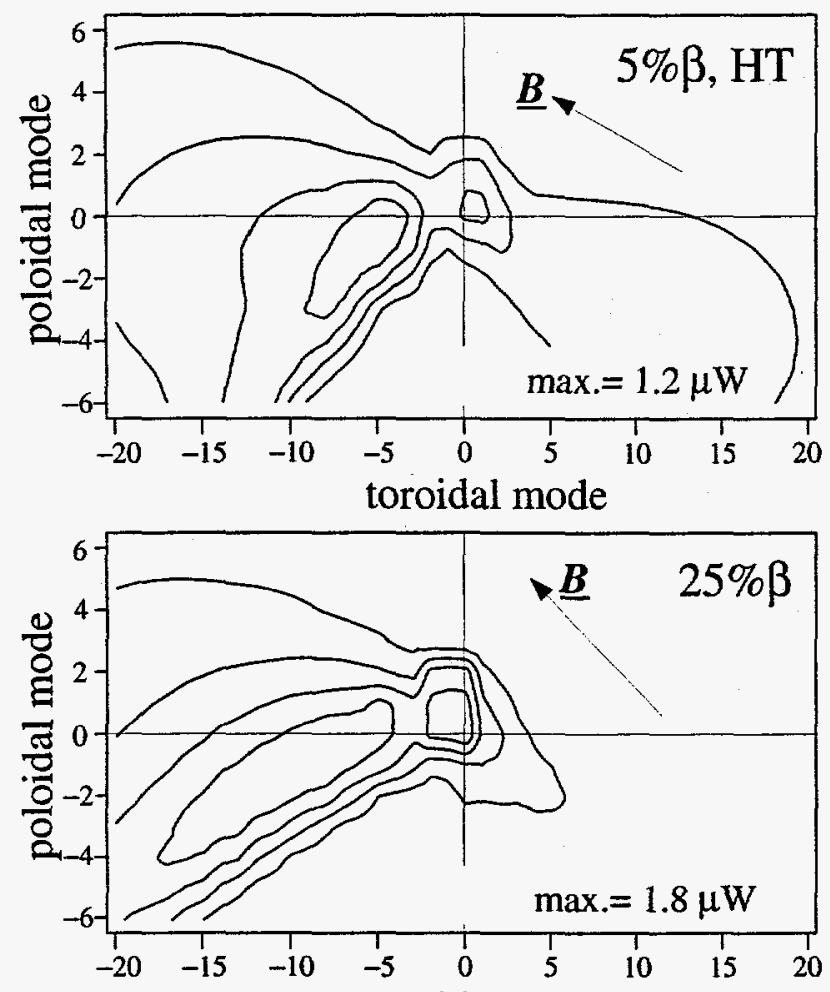

toroidal mode

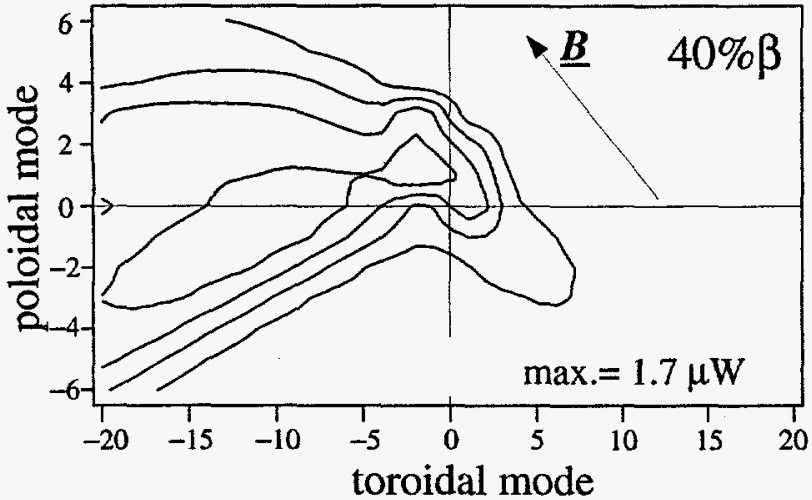

toroidal mode
Impulse source, $24^{\circ}$ tilt
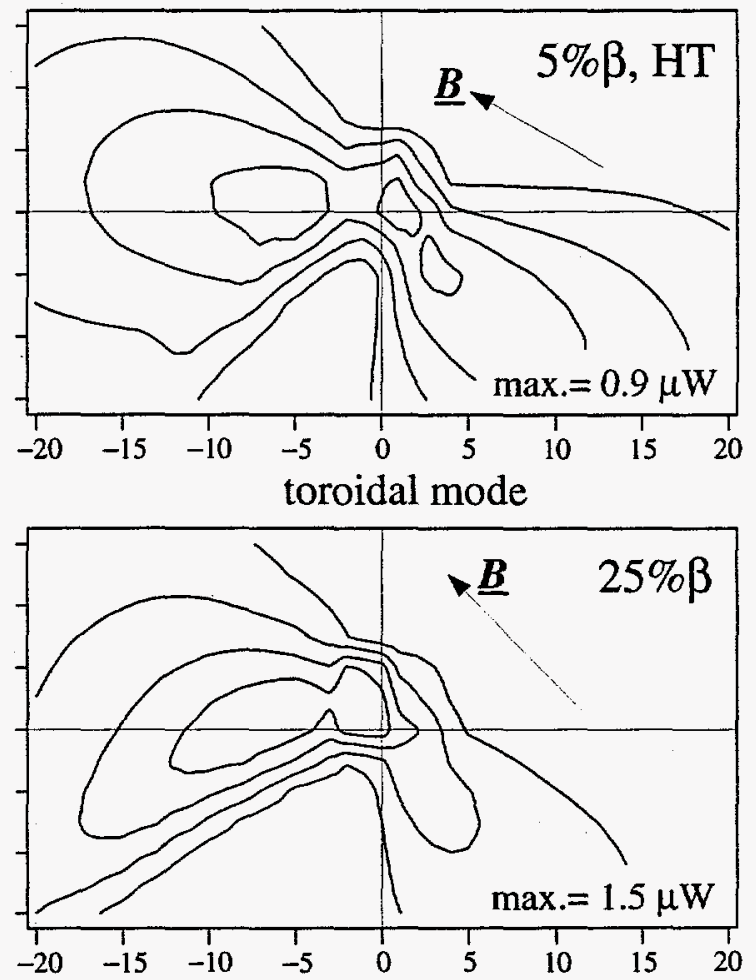
toroidal mode

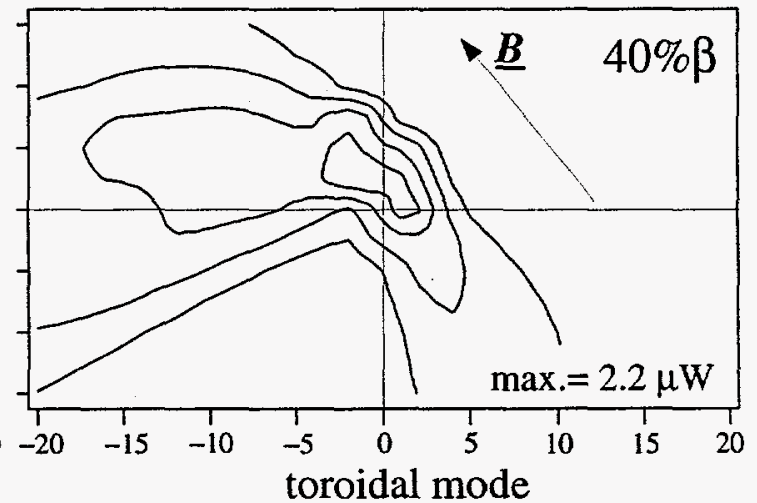

Fig. 3. Contours of constant power for $20 \%, 40 \%, 60 \%$, and $80 \%$ of the maximum power indicated in each frame ( 1 amp of current in each strap). To reduce the noise caused by surface modes, the loading data have been smoothed by averaging nearest neighbors in a single pass. 


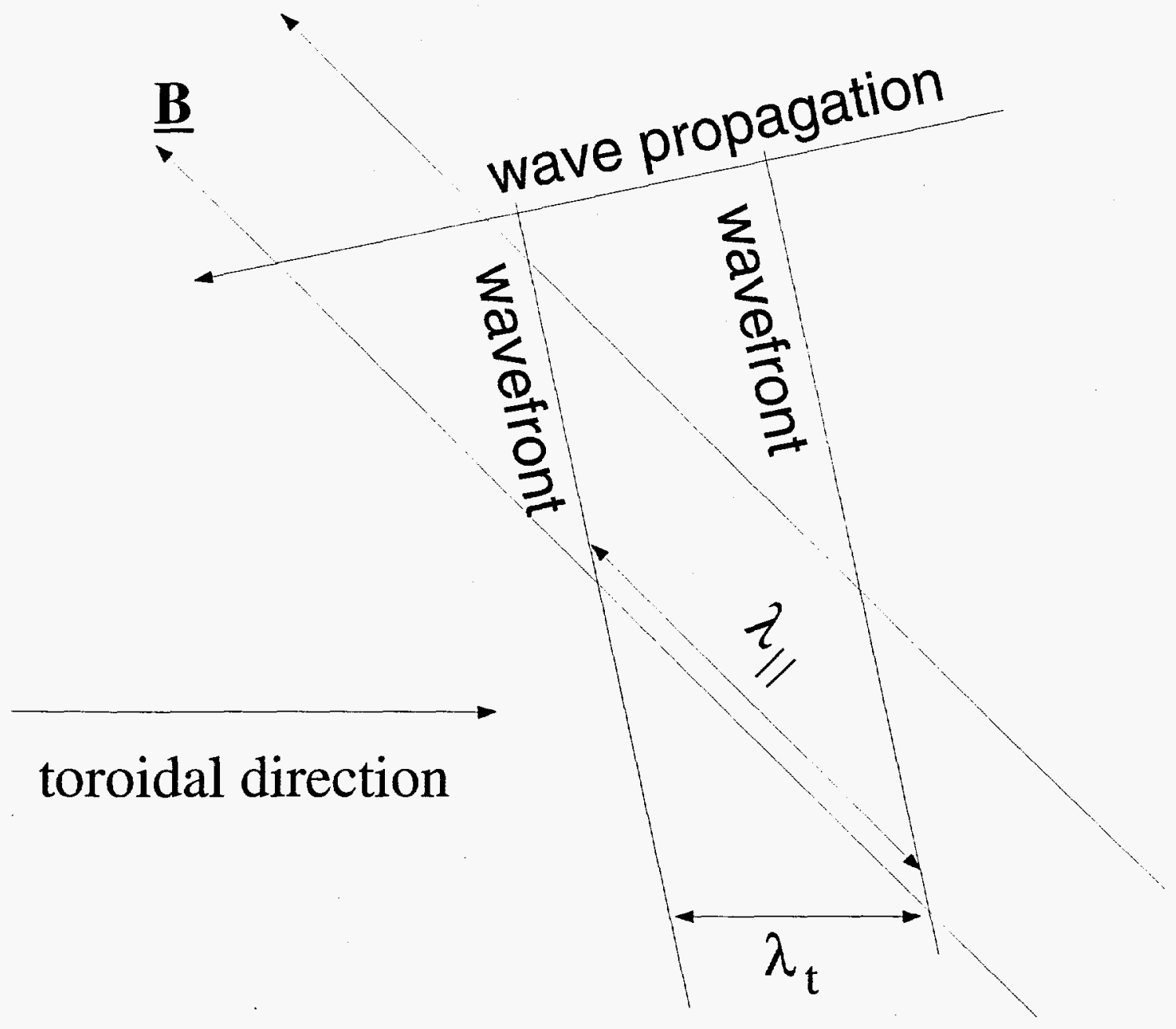

Fig. 4. A large poloidal magnetic field can influence the poloidal Fourier modes for optimal coupling. The toroidal wavelength is determined by the antenna phasing, so modes propagating at a downward angle can have longer parallel wavelengths and better radial propagation in the edge than the modes propagating upward. 


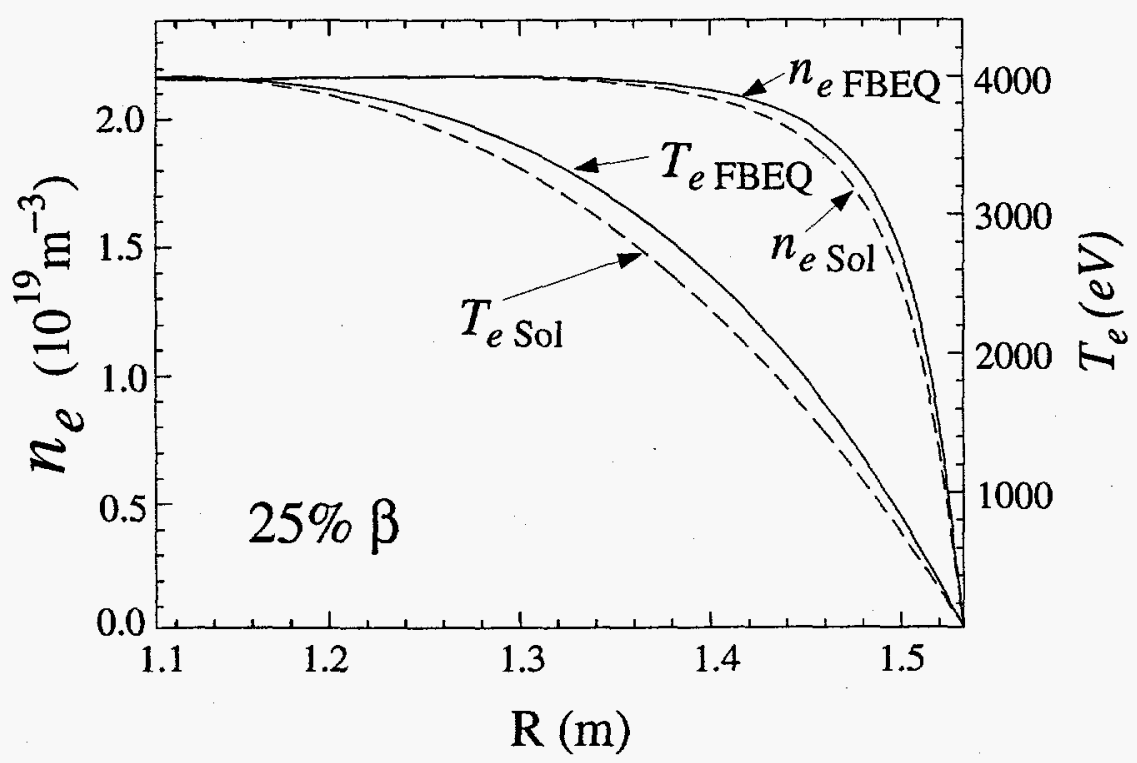

Fig. 5. The density and temperature profiles can be made very nearly equal for both the FBEQ and Solov'ev equilibria for the $25 \% \beta$ case.
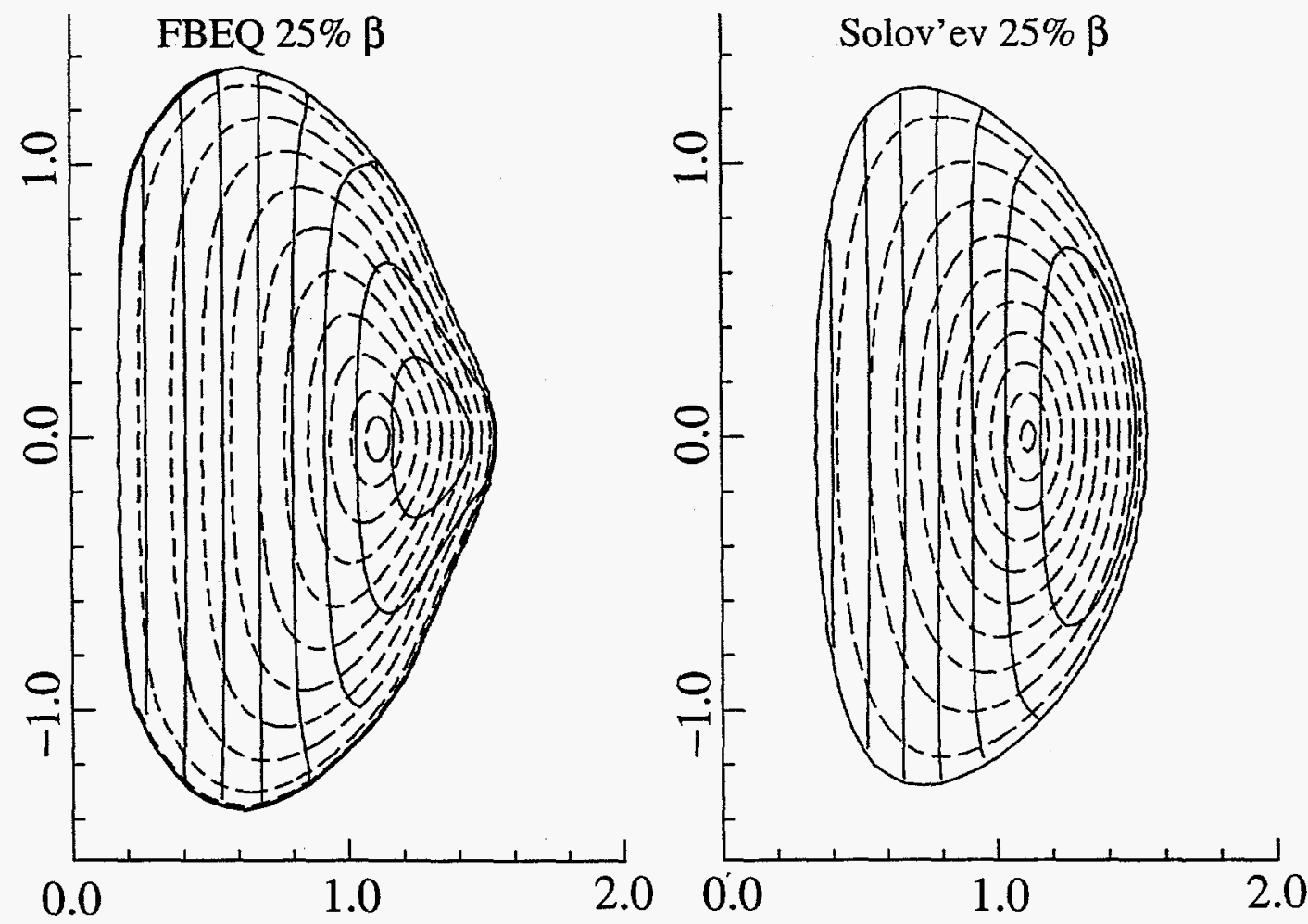

Fig. 6. The magnetic topology for the FBEQ and Solov'ev equilibria are similar, but finite $\beta$ effects modify the magnetic field strength and trapped particle fraction for the more accurate FBEQ result for the $25 \% \beta$ case. 


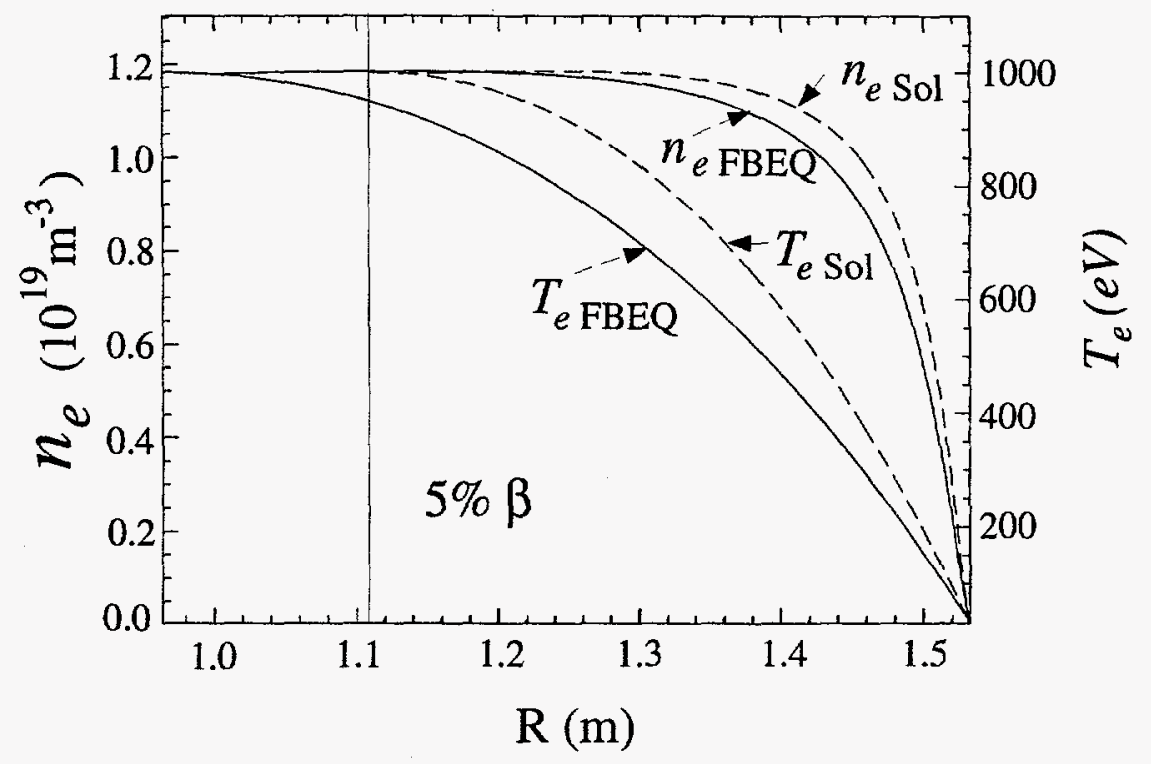

Fig. 7. The comparison between the FBEQ and Solov'ev equilibria is more difficult at $5 \%$ $\beta$ than at $25 \% \beta$ because the Solov'ev model does not include triangularity needed to properly locate the magnetic axis.
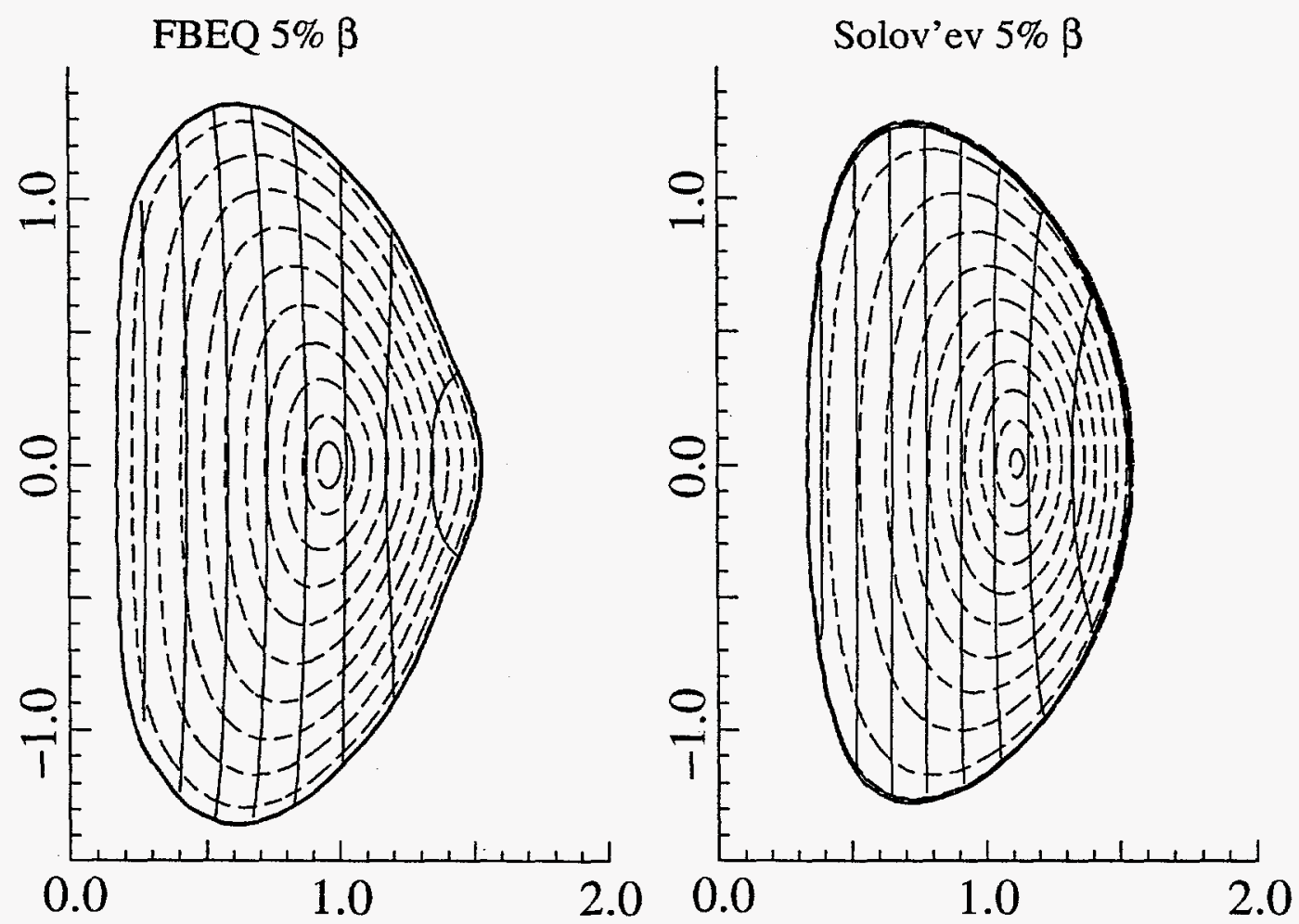

Fig. 8. The magnetic topology for the FBEQ and Solov'ev equilibria are similar for the $5 \% \beta$ case except for the location of the magnetic axis. The contours of constant $|\mathrm{B}|$ are not strongly affected by the finite $\beta$. 


\section{FBEQ}
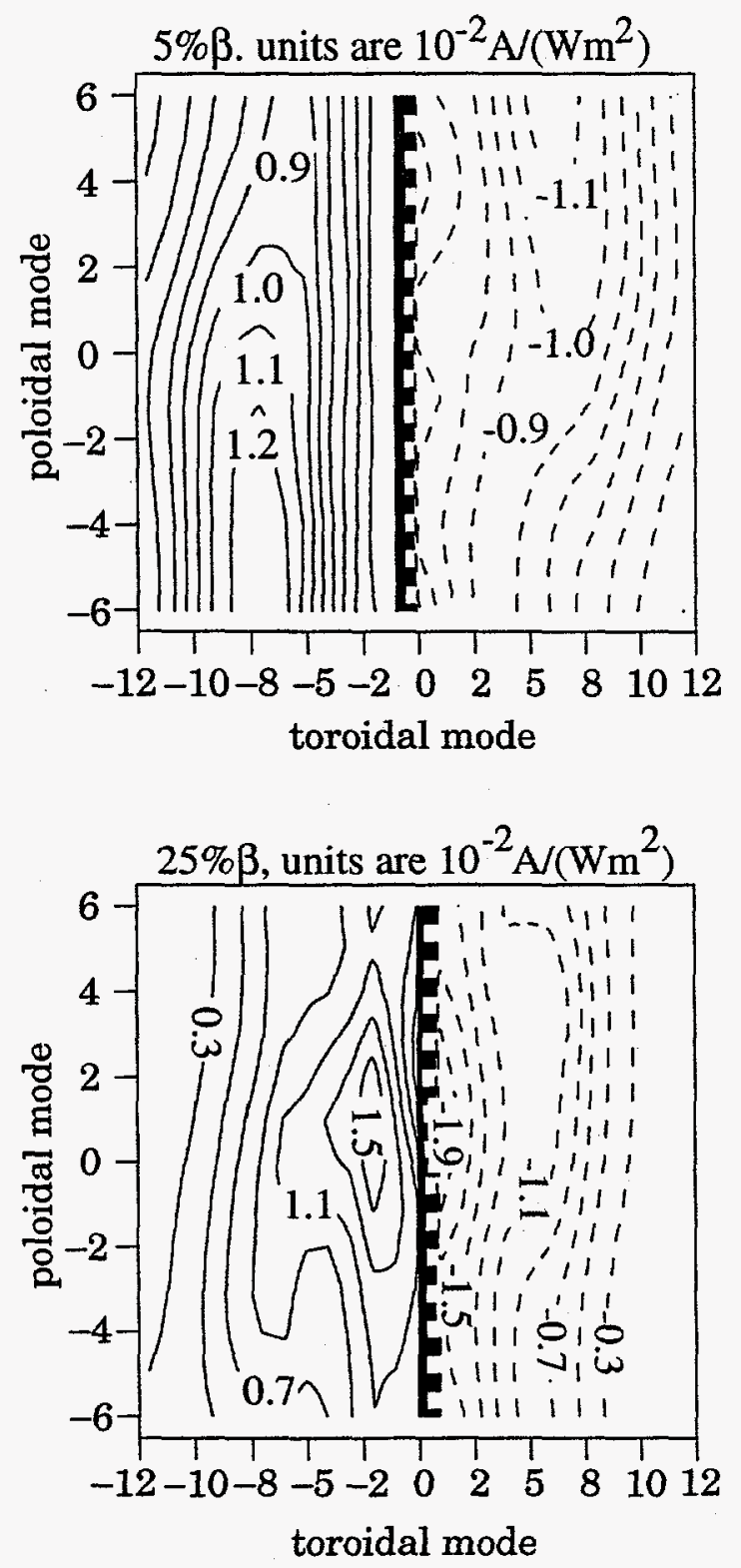

\section{Solov'ev}
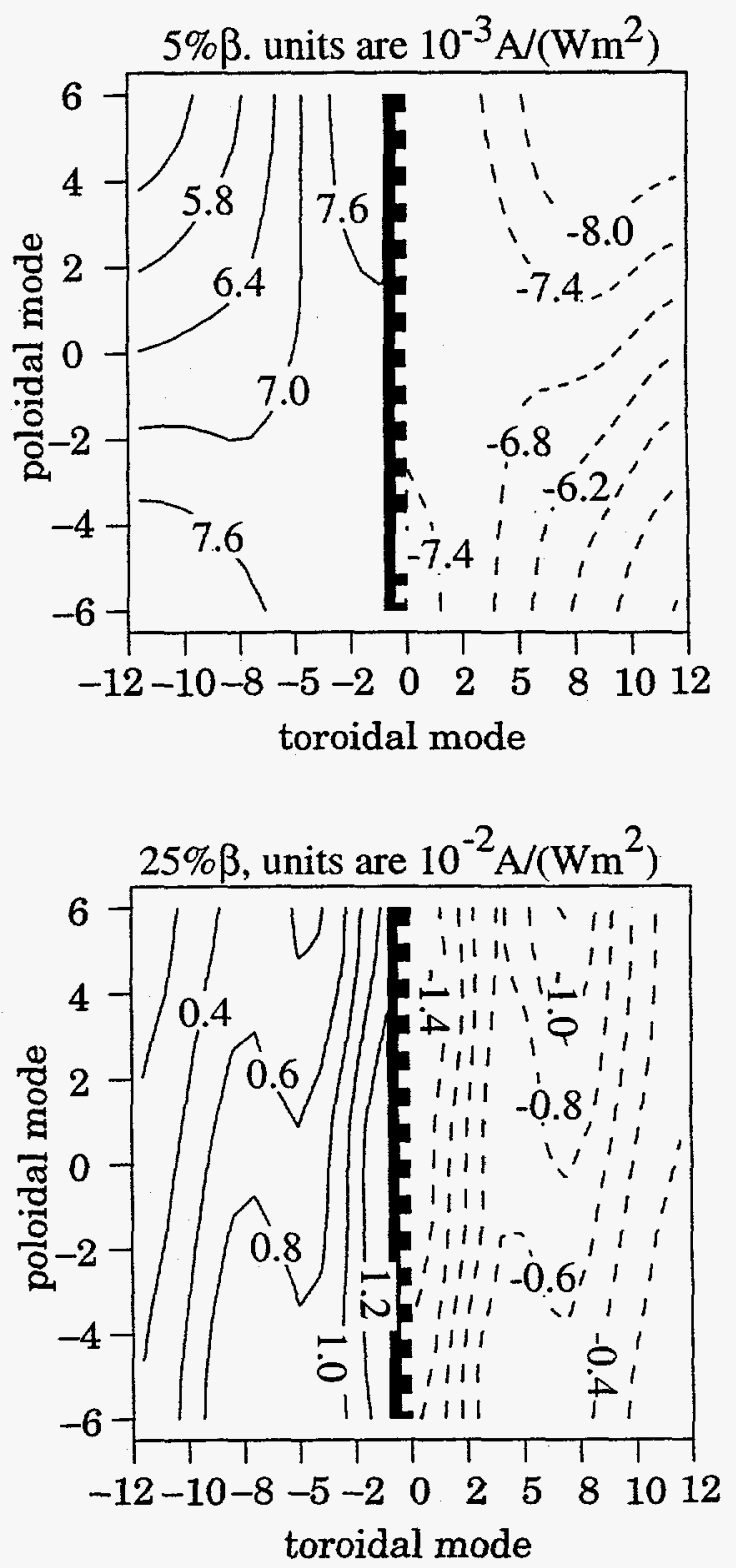

Fig. 9. Contours of HHFW current drive efficiency for fixed poloidal and toroidal number on the last closed flux surface provide a target for the antenna design. The poloidal asymmetry caused by density gradients and the poloidal field leads to a poloidal shift in the launched modes required for optimum efficiency. Finite $\beta$ effects in the $25 \% \beta$ case lead to a reduction of trapped electron effects for the fastest toroidal mode numbers. Note the sensitivity of the results to the plasma equilibrium. 

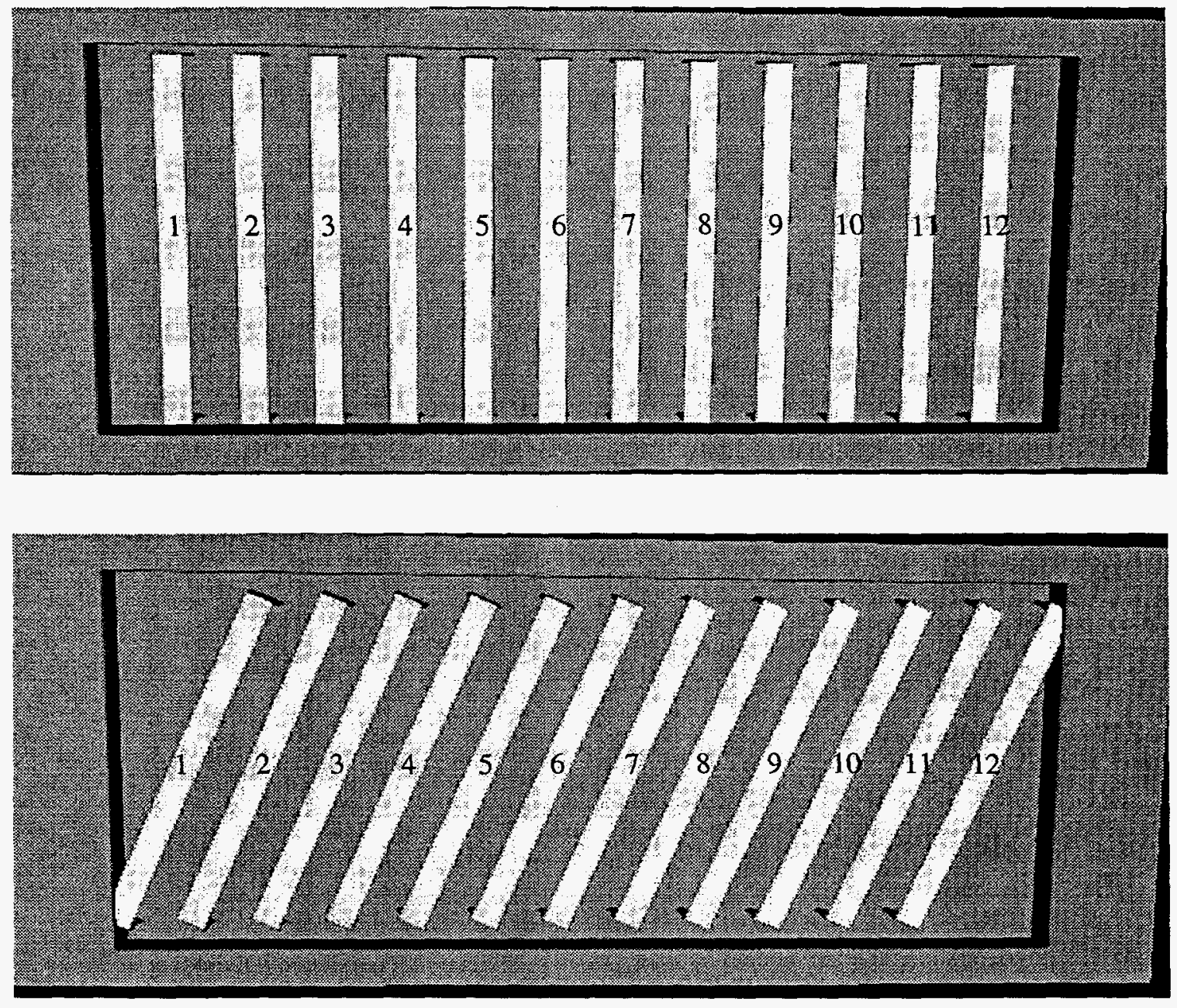

Fig. 10. The RANT3D model for poloidally oriented $\left(\theta=90^{\circ}\right)$ and tilted $\left(\theta=66^{\circ}\right)$ straps is used to examine the effect of tilting the current straps for 1 MA discharges in NSTX. The conducting poloidal limiters are roughly placed at the location of the stabilizing plates. The toroidal limiters are located somewhat arbitrarily but do not affect many of the results. 

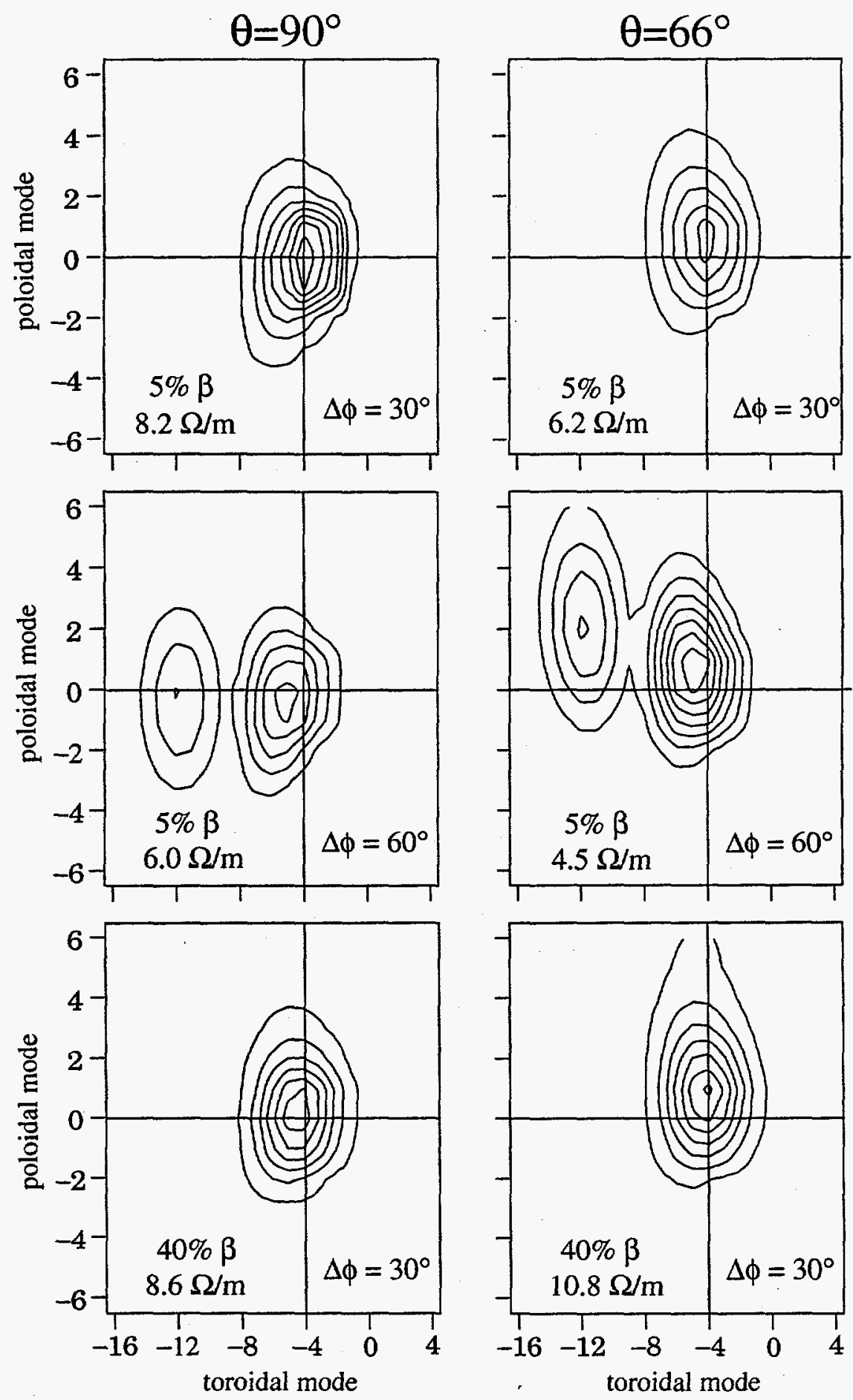

Fig. 11. Tilting the current straps produces a poloidal shift in the launched power spectrum. This shift is helpful to the loading for high plasma $\beta$ because of the improved . polarization, but tilting is detrimental to loading at low $\beta$ because of the evanescent layer. 

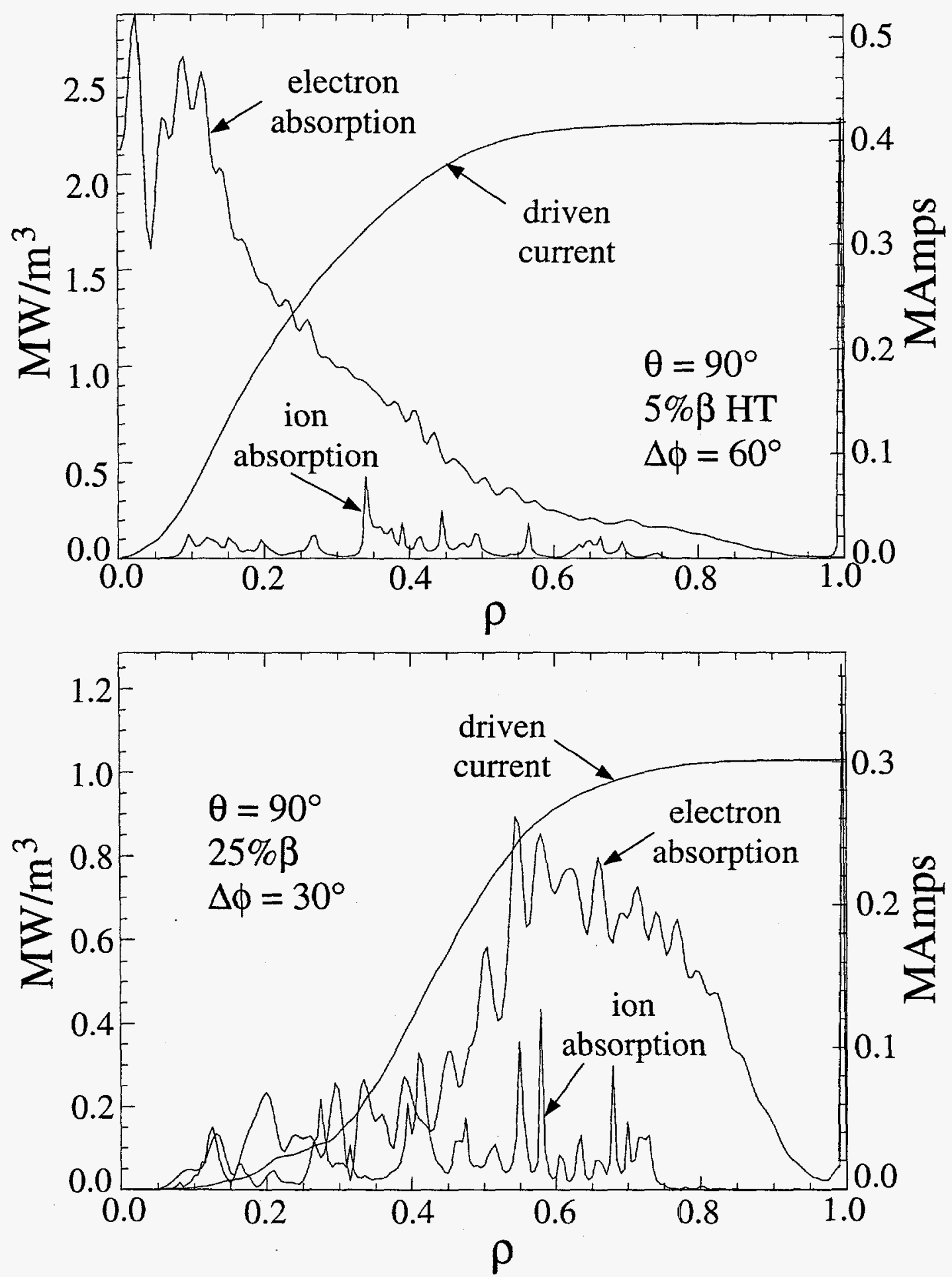

Fig. 12. The power and current profiles can be estimated by using fields from the RANT code as boundary conditions for the PICES code. At $5 \% \beta$, the waves penetrate into the center of the machine, but power is absorbed off-axis for the $25 \% \beta$ case. 

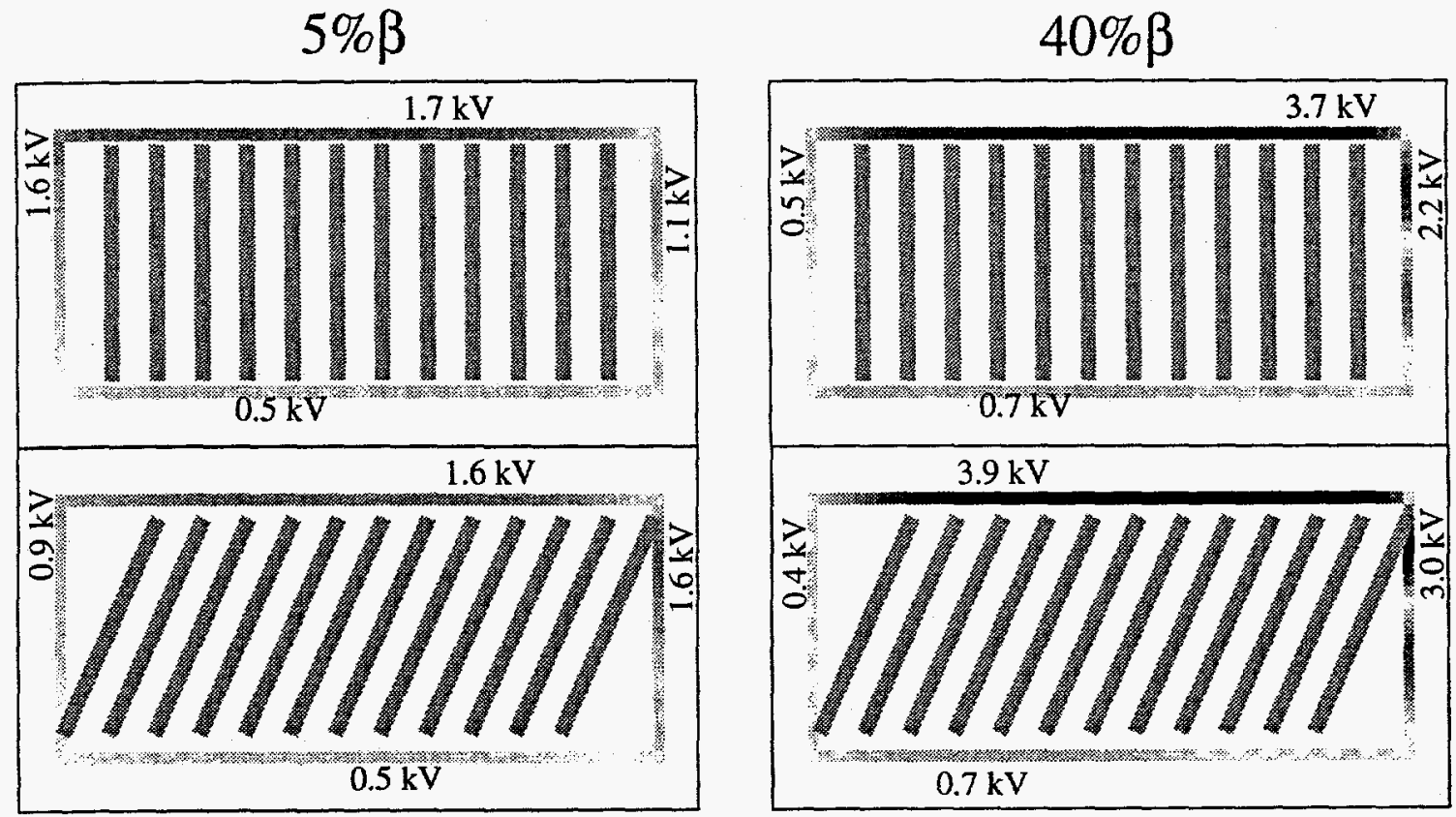

Fig. 13. The upper stabilizing plate encounters the largest RF rectified sheaths for all antenna geometries. The rectified potential is estimated by integrating the electrostatic portion of the parallel electric field along a field line near the sharp boundary of the conducting limiters. The estimate for the rectified potential is much higher for the $40 \% \beta$ case than for the $5 \% \beta$ case, and could reach $\sim 4 \mathrm{kV}$ for $6 \mathrm{MW}$ of power in the $40 \% \beta$ case. Tilting the straps does not help to reduce the voltage significantly. 
41. S. Moriyama, Japan Atomic Energy Research Institute, Naka Fusion Research Establishment, Mukoyama 801-1, Naka-machi, Naka-gun, Ibaraki-ken 311-01, JAPAN.

42. F. Najmabadi, University of California, San Diego, Fusion Energy Research Program, Mail Code 0417, 9500 Gilman Drive, La Jolla, CA 92093-0417.

43. G. A. Navratil, Columbia University, Department of Applied Physics, 202 S.W. Mudd Bldg., MC 4701, New York, NY 10027.

44. M. O'Brien, UKAEA Fusion, Culham, Abingdon, Oxfordshire, OX14 3DB, UNITED KINGDOM.

45. E. Oktay, Office of Fusion Energy Sciences, Office of Energy Research, ER-55, U.S. Department of Energy, 19901 Germantown Road, Germantown, MD 20874-1290.

46. M. Ono, Princeton Plasma Physics Laboratory, P. O. Box 451, Princeton, NJ 08543-0451.

47. A. L. Opdenaker, Office of Fusion Energy Sciences, Office of Energy Research, ER-52, U.S. Department of Energy, 19901 Germantown Road, Germantown, MD 20874-1290.

48. M. Porkolab, Massachusetts Institute of Technology, Room NW16-288, 167 Albany St., Cambridge, MA 02139.

49. S. C. Prager, Chamberlin Hall, University of Wisconsin, 1150 University Avenue, Madison, WI 53706-1390.

50. W. A. Sadowski, Office of Fusion Energy Sciences, Office of Energy Research, ER-55, U.S. Department of Energy, 19901 Germantown Road, Germantown, MD 20874-1290.

51. T. Simonen, General Atomics, P.O. Box 85608, San Diego, CA 92186-5608.

52. K. I. Thomassen, Lawrence Livermore National Laboratory, P.O. Box 808 (L-637), Livermore, CA 94551-9900.

53. J. W. Willis, Office of Fusion Energy Sciences, Office of Energy Research, ER-55, U.S. Department of Energy, 19901 Germantown Road, Germantown, MD 20874-1290.

54. J. R. Wilson, Princeton Plasma Physics Laboratory, P. O. Box 451, Princeton, NJ 08543-0451.

55. H. E. Clark, DOE-ORO, ORNL, 4500 N, MS-6269.

56-57. DOE Offiee of Scientific and Technical Information, P. O. Box 62, Oak Ridge, TN 37831-0062. 

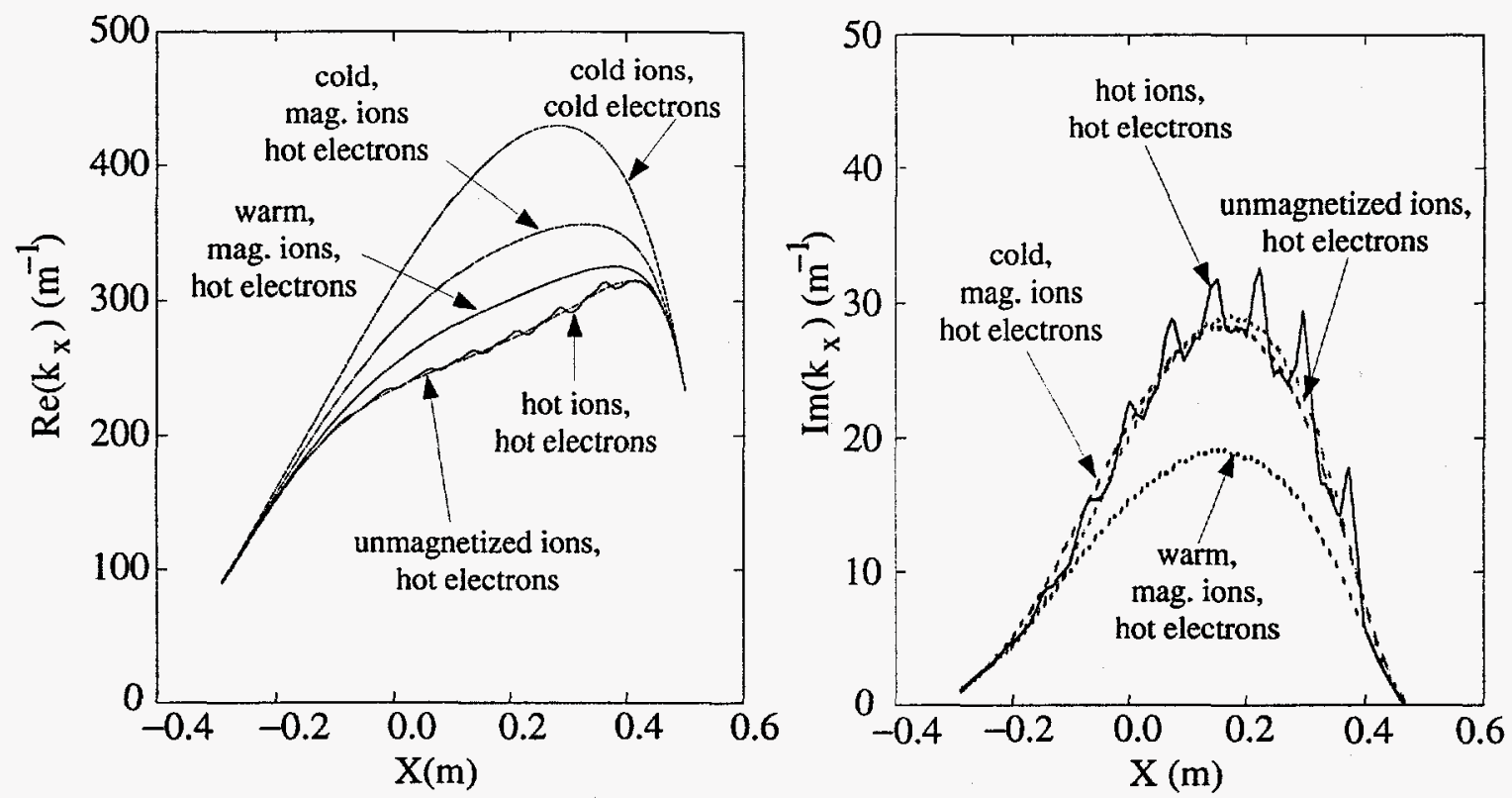

Fig. 14. The validity of various approximations for the plasma conductivity is found by comparing the real and imaginary part of the radial wave number. The peaks in the curve for the hot magnetized ion calculation are caused by various high order harmonics; a maximum of 40 were retained. The best approximation to the hot ion $\beta$ result uses unmagnetized ions. The warm plasma approximation underestimates the damping by the ions for these plasma parameters: $n=12,41 \mathrm{MHz}, \mathrm{B}(0)=0.32 \mathrm{~T}, \mathrm{n}_{\mathrm{e}}(0)=5 \times 10^{19} \mathrm{~m}^{-3}$, and $\mathrm{T}_{\mathrm{e}}(0)=\mathrm{T}_{\mathrm{i}}(0)=2 \mathrm{KeV}$.
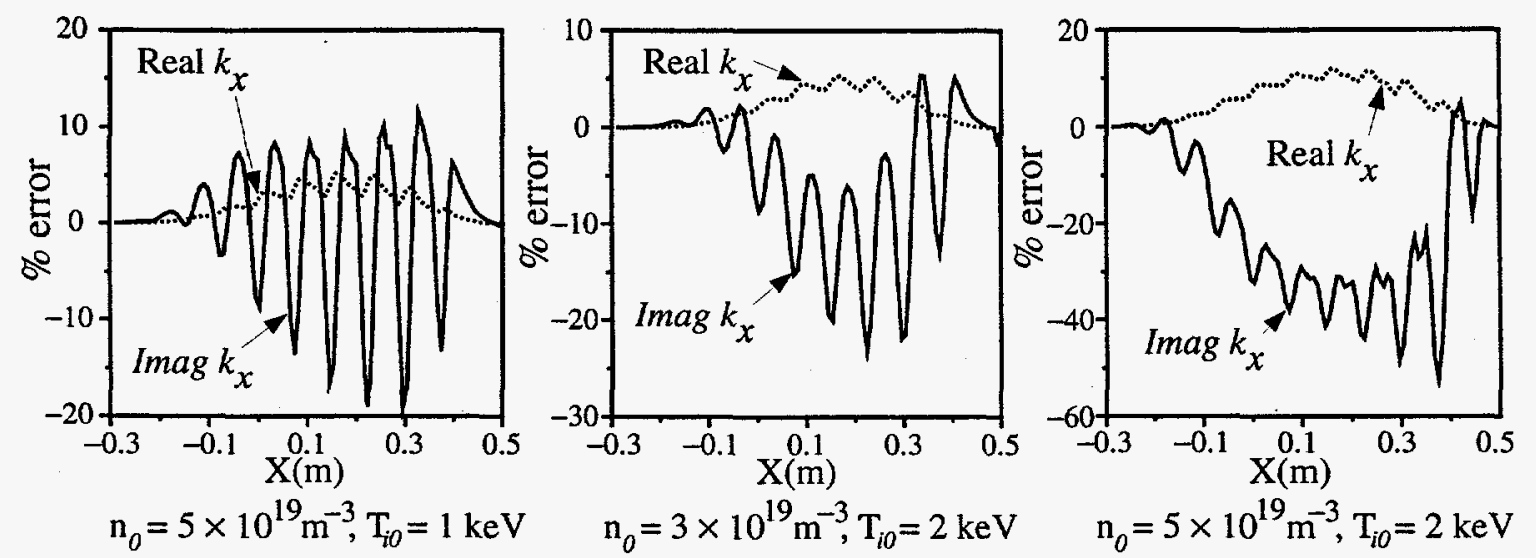

Fig. 15. The error relative to the hot ion calculation with 40 harmonics shows that the warm ion approximation remains reasonable for $\beta_{i}<12 \%$ in NSTX at $41 \mathrm{MHz}$; $\mathrm{Te}(0)=2 \mathrm{KeV}$ for all cases. 

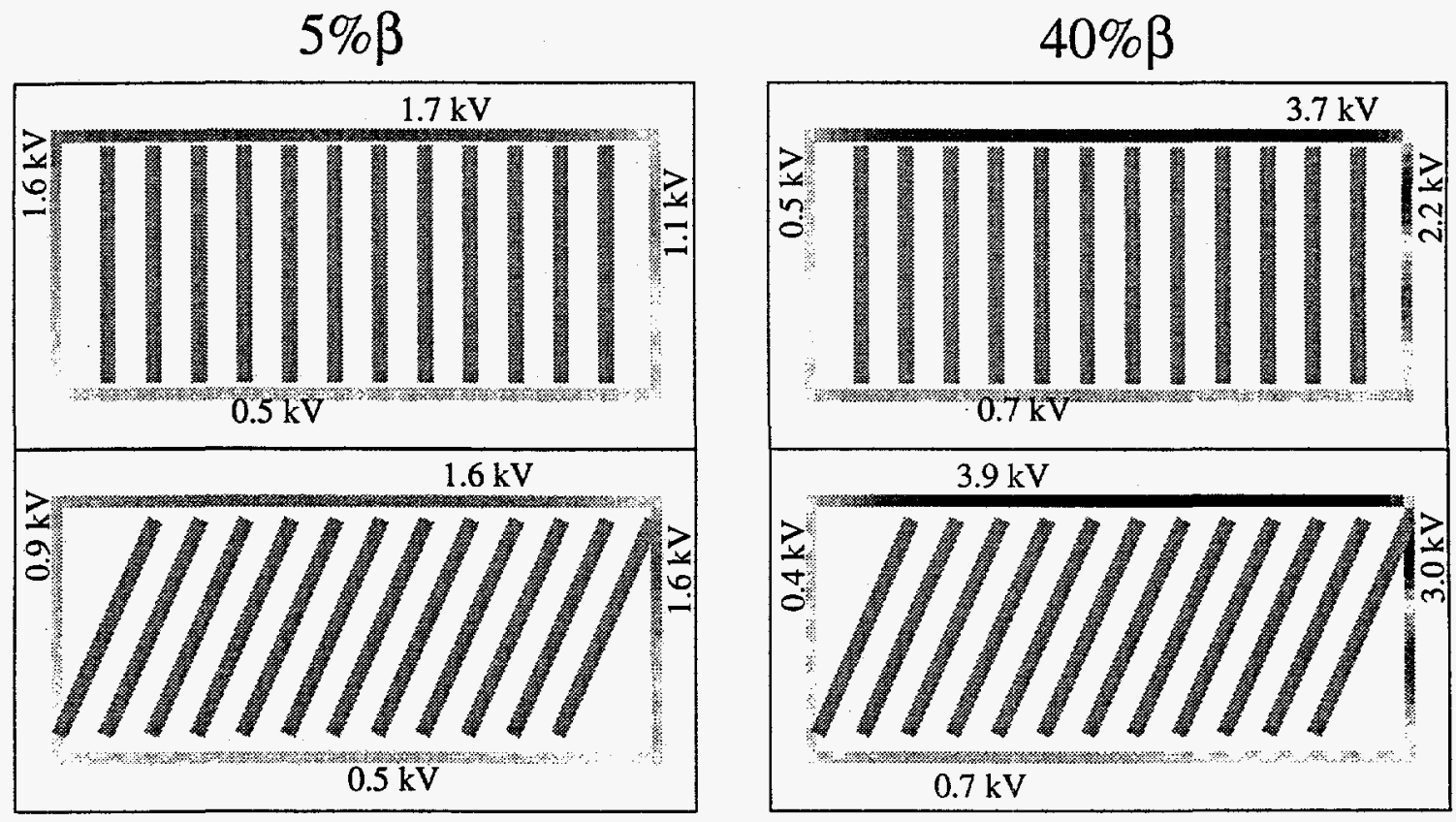

Fig. 13. The upper stabilizing plate encounters the largest RF rectified sheaths for all antenna geometries. The rectified potential is estimated by integrating the electrostatic portion of the parallel electric field along a field line near the sharp boundary of the conducting limiters. The estimate for the rectified potential is much higher for the $40 \% \beta$ case than for the $5 \% \beta$ case, and could reach $\sim 4 \mathrm{kV}$ for $6 \mathrm{MW}$ of power in the $40 \% \beta$ case. Tilting the straps does not help to reduce the voltage significantly. 


\section{INTERNAL DISTRIBUTION}

1. D. B. Batchelor

2. L. A. Berry

3. B. A. Carreras

4-8. M. D. Carter

9-10. E. F. Jaeger

11. S. L. Milora

12. P. K. Mioduszewski

13. Y-K. M. Peng
14. P. M. Ryan

15. M. J. Saltmarsh

16. D. J. Strickler

17. D. W. Swain

18. Fusion Energy Division Library

19. ETD/FED Publications Office

20. Laboratory Records Department - RC

21. Central Research Library

\section{EXTERNAL DISTRIBUTION}

22. N. Anne Davies, Associate Director for Fusion Energy Sciences, Office of Energy Research, ER-50, U.S. Department of Energy, 19901 Germantown Road, Germantown, MD 20847-1290.

23. Martha A. Krebs, Director, Office of Energy Research, ER-1, U.S. Departmernt of Energy, Headquarters, Forrestal Building, 1000 Independence Avenue, SW, Washington, DC 20585.

24. E. G. Cumesty, ORNL Site Manager, U.S. Department of Energy, Oak Ridge Operations, P. O. Box 2008, Oak Ridge, TN 37831-6269.

25. B. Beaumont, DRFC, Association Euratom-CEA, C.E. Cadarache, 13108 Saint Paul-Lez-Durance, FRANCE.

26. R. A. Blanken, Office of Fusion Energy Sciences, Office of Energy Research, ER-55, U.S. Department of Energy, 19901 Germantown Road, Germantown, MD 20874-1290.

27. C. W. Bolton, Office of Fusion Energy Sciences, Office of Energy Research, ER-52, U.S. Department of Energy, 19901 Germantown Road, Germantown, MD 20874-1290.

28. J. D. Callen, Engineering Research Building, University of Wisconsin, 1500 Johnson Drive, Madison, WI 53706-1687.

29. R. Cesario, ENEA-Dipartimento Energia, Centro Ricerche di Frascati, Roma, ITALY.

30. M. D. Crisp, Office of Fusion Energy Sciences, Office of Energy Research, ER- 55, U.S. Department of Energy, 19901 Germantown Road, Germantown, MD 20874-1290.

31. R. Y. Dagazian, Office of Fusion Energy Sciences, Office of Energy Research, ER-52, U.S. Department of Energy, 19901 Germantown Road, Germantown, MD 20874-1290.

32. S. A. Eckstrand, Office of Fusion Energy Sciences, Office of Energy Research, ER-52, U.S. Department of Energy, 19901 Germantown Road, Germantown, MD 20874-1290.

33. R. Galvao, Instituto de Física da Universidade de São Paulo, P.O. Box 66318, São Paulo CEP05315-970, BRAZIL.

34. T. V. George, Office of Fusion Energy Sciences, Office of Energy Research, ER-52, U.S. Department of Energy, 19901 Germantown Road, Germantown, MD 20874-1290.

35. V.K. Gusev, A.F. Ioffe Physico-Technical Institute, 194021, Politechnicheskaya st. 26, S. Petersburg, RUSSIA.

36. J. Jacquinot, JET Joint Undertaking, Abingdon, Oxfordshire, OX14 3EA, UNITED KINGDOM.

37. M. D. Johnson, Office of Fusion Energy Sciences, Office of Energy Research, ER-52, U.S. Department of Energy, 19901 Germantown Road, Germantown, MD 20874-1290.

38. S. M. Kaye, Princeton Plasma Physics Laboratory, P. O. Box 451, Princeton, NJ 08543-0451.

39. R. Majeski, Princeton Plasma Physics Laboratory, P. O. Box 451, Princeton, NJ 08543-0451.

40. R. H. McKnight, Office of Fusion Energy Sciences, Office of Energy Research, ER-55, U.S. Department of Energy, 19901 Germantown Road, Germantown, MD 20874-1290. 
41. S. Moriyama, Japan Atomic Energy Research Institute, Naka Fusion Research Establishment, Mukoyama 801-1, Naka-machi, Naka-gun, Ibaraki-ken 311-01, JAPAN.

42. F. Najmabadi, University of California, San Diego, Fusion Energy Research Program, Mail Code 0417, 9500 Gilman Drive, La Jolla, CA 92093-0417.

43. G. A. Navratil, Columbia University, Department of Applied Physics, 202 S.W. Mudd Bldg., MC 4701, New York, NY 10027.

44. M. O'Brien, UKAEA Fusion, Culham, Abingdon, Oxfordshire, OX14 3DB, UNITED KINGDOM.

45. E. Oktay, Office of Fusion Energy Sciences, Office of Energy Research, ER-55, U.S. Department of Energy, 19901 Germantown Road, Germantown, MD 20874-1290.

46. M. Ono, Princeton Plasma Physics Laboratory, P. O. Box 451, Princeton, NJ 08543-0451.

47. A. L. Opdenaker, Office of Fusion Energy Sciences, Office of Energy Research, ER-52, U.S. Department of Energy, 19901 Germantown Road, Germantown, MD 20874-1290.

48. M. Porkolab, Massachusetts Institute of Technology, Room NW16-288, 167 Albany St., Cambridge, MA 02139.

49. S. C. Prager, Chamberlin Hall, University of Wisconsin, 1150 University Avenue, Madison, WI 53706-1390.

50. W. A. Sadowski, Office of Fusion Energy Sciences, Office of Energy Research, ER-55, U.S. Department of Energy, 19901 Germantown Road, Germantown, MD 20874-1290.

51. T. Simonen, General Atomics, P.O. Box 85608, San Diego, CA 92186-5608.

52. K. I. Thomassen, Lawrence Livermore National Laboratory, P.O. Box 808 (L-637), Livermore, CA 94551-9900.

53. J. W. Willis, Office of Fusion Energy Sciences, Office of Energy Research, ER- 55, U.S. Department of Energy, 19901 Germantown Road, Germantown, MD 20874-1290.

54. J. R. Wilson, Princeton Plasma Physics Laboratory, P. O. Box 451, Princeton, NJ 08543-0451.

55. H. E. Clark, DOE-ORO, ORNL, 4500N, MS-6269.

56-57. DOE Offiee of Scientific and Technical Information, P. O. Box 62, Oak Ridge, TN 37831-0062. 